\title{
L $^{\infty}$-NORM MINIMAL CONTROL OF THE WAVE EQUATION: ON THE WEAKNESS OF THE BANG-BANG PRINCIPLE
}

\author{
Martin Gugat $^{1}$ And Gunter Leugering ${ }^{1}$
}

\begin{abstract}
For optimal control problems with ordinary differential equations where the $L^{\infty}$-norm of the control is minimized, often bang-bang principles hold. For systems that are governed by a hyperbolic partial differential equation, the situation is different: even if a weak form of the bang-bang principle still holds for the wave equation, it implies no restriction on the form of the optimal control. To illustrate that for the Dirichlet boundary control of the wave equation in general not even feasible controls of bang-bang type exist, we examine the states that can be reached by bang-bang-off controls, that is controls that are allowed to attain only three values: Their maximum and minimum values and the value zero. We show that for certain control times, the difference between the initial and the terminal state can only attain a finite number of values. For the problems of optimal exact and approximate boundary control of the wave equation where the $L^{\infty}$-norm of the control is minimized, we introduce dual problems and present the weak form of a bang-bang principle, that states that the values of $L^{\infty}$-norm minimal controls are constrained by the sign of the dual solutions. Since these dual solutions are in general given as measures, this is no restriction on the form of the control function: the dual solution may have a finite support, and when the dual solution vanishes, the control is allowed to attain all values from the interval between the two extremal control values.
\end{abstract}

Mathematics Subject Classification. 49K20,35L05

Received December 7, 2005. Revised March 6 and September 11, 2006.

Published online September 21, 2007.

\section{INTRODUCTION}

For finite dimensional linear systems, the Kalman condition guarantees that a control of minimal $L^{\infty}$-norm is of bang-bang form (see $[17,20]$ and 1.1 below). For distributed parameter systems, the situation is more involved. For the heat equation, where analytic solutions appear, a bang-bang structure in a weak form in the sense of Krabs (see [14], Ths. 2.4.13 and 2.4.14) and Seidman (see [21]) remains. In this paper we try to illuminate the situation for systems governed by the 1-D wave equation, where a finite wave speed is essential. We show that the weak form of the bang-bang principle which holds for the wave equation does not imply any restriction on the form of the optimal controls.

\footnotetext{
Keywords and phrases. Optimal control of pdes, optimal boundary control, wave equation, bang-bang, bang-bang-off, dual problem, dual solutions, $L^{\infty}$, measures.

${ }^{1}$ Lehrstuhl 2 für Angewandte Mathematik, Martensstr. 3, 91058 Erlangen, Germany;

gugat@am.uni-erlangen.de; leugering@am.uni-erlangen.de
} 


\subsection{About the bang-bang principle}

Let $Y$ be a separable Banach space that is used as the space for the controls. We consider pointwise timedependent control constraints of the form $u(t) \in U(t)$ almost everywhere on the time interval $[0, T]$ that are given by a multifunction $U: t \mapsto U(t)$ where for all $t \in[0, T]$, the set $U(t) \subset Y$ is nonempty and closed. The analysis for the infinite-dimensional case is based upon measurable multifunctions.

Definition 1.1. A multifunction $U$ with $U(t)$ nonempty and closed for all $t \in[0, T]$ is said to be measurable, if for every $y \in Y$, the function $t \mapsto \inf _{\nu \in U(t)}\|y-\nu\|$ is measurable. We define the admissible set $\mathcal{U}_{U}^{T}$ of controls

$$
\mathcal{U}_{U}^{T}:=\left\{u \in L^{1}(0, T ; Y): u(t) \in U(t) \text { a.e. on }(0, T)\right\}
$$

\subsubsection{The finite-dimensional case}

For all $t \geq 0$, let $U(t) \subset \mathbf{R}^{m}$ be a nonempty closed set and let $U(\cdot)$ be a measurable multifunction. Let us consider first the classical finite dimensional linear control problem

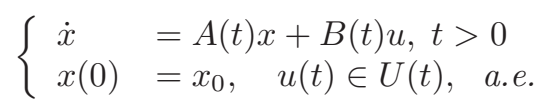

where $x \in \mathbf{R}^{n}, A(\cdot) \in L_{\text {loc }}^{1}\left(\mathbf{R}_{+} ; \mathbf{R}^{n \times n}\right), B(\cdot) \in L_{\text {loc }}^{1}\left(\mathbf{R}_{+} ; \mathbf{R}^{n \times m}\right)$. It is well-known that under these circumstances there exists a unique absolutely continuous solution $x(\cdot)$ of $(2)$.

Definition 1.2. Let $Y=\mathbf{R}^{m}$ and $X=\mathbf{R}^{n}$. We define the attainable set

$$
\mathcal{R}_{U}^{T}\left(x_{0}\right):=\left\{z \in X: \text { There exists } u \in \mathcal{U}_{U}^{T} \text { such that } x(\cdot) \text { solves }(2) \text { and satisfies } x(T)=z\right\}
$$

as the set of points $z$ that can be reached from the initial datum $x_{0}$ in time $T>0$ by the action of the control $u$.

It is known that

$$
\overline{\operatorname{conv}} \mathcal{U}_{U}^{T}=\mathcal{U}_{\overline{\operatorname{conv}} U}^{T}
$$

At this point recall the definition of extreme points:

Definition 1.3. Let $V$ be a vector space and $A \subset V$ a set. A point $a \in A$ is called an extreme point of $A$ if for any two points $b, c \in A$ such that $(b+c) / 2=a$ the equation $b=c=a$ holds. The set of all extreme points of $A$ is denoted ext $A$.

If the sets $U(t)$ are convex and compact, one has the bang-bang principle

$$
\mathcal{R}_{U}^{T}\left(x_{0}\right)=\mathcal{R}_{\operatorname{ext} U}^{T}\left(x_{0}\right)
$$

which states that each attainable point can also be obtained by controls that satisfy $u(t) \in \operatorname{ext} U(t)$ a.e., see Hermes and Lasalle [11], Lee and Markus [16] as basic references. The bang-bang principle states that such controls can be realized by functions with values in the extreme points of $U(t)$. In particular, if for all $t$ the set $U(t)=H$ is the same set $H$ and $H$ is a hypercube in $\mathbf{R}^{m}$, then the extremal controls switch between the corners of that cube. In intervals in $Y=\mathbf{R}^{1}$ there are only two extremal values, therefore the name 'bang-bang'. This principle has become a paradigm in control theory and in applied sciences, as it states that one may as well control in fully exploring all resources any time in the sense that at any time, the control contraints are active. For example, if $U(t)=[-1,1]$ the absolute value $|u(t)|$ can be chosen to be equal to one at any time. This will become even more important in optimal control problems. 


\subsubsection{The infinite-dimensional case}

Let $X=Y$ be a separable Banach-space. For all $t \geq 0$, let $U(t) \subset X$ be a nonempty closed set and let $U(\cdot)$ be a measurable multifunction. Define the reachable set as in Definition 1.2 with the infinite-dimensional space $X=Y$. For all $t \geq 0$, let $A(t)$ be an unbounded linear operator in $X$ and let $B(t) \in \mathcal{L}(X)$ be a bounded operator, where $\mathcal{L}(X)$ denotes the space of bounded operators in $X$. Assume that $B(\cdot) \in L_{\text {loc }}^{\infty}\left(\mathbf{R}_{+} ; \mathcal{L}(X)\right)$. Using Kato's theory [13] we may consider solutions of the distributed parameter system

$$
\begin{cases}\dot{x} & =A(t) x+B(t) u, t>0 \\ x(0) & =x_{0}, \quad u(t) \in U(t) \quad \text { a.e. } \\ u(\cdot) & \in L^{1}(0, T ; X) .\end{cases}
$$

We assume that the homogeneous problem

$$
\begin{cases}\dot{x} & =A(t) x, t>0 \\ x(0) & =x_{0}\end{cases}
$$

admits a fundamental solution i.e. an evolution operator

$$
\Phi(\cdot, \cdot):\{(t, s): 0 \leq s \leq t\} \rightarrow \mathcal{L}(X)
$$

See Kato [12]. In fact, for time-independent operators $A(t)=A$ the fundamental solution is given by the $C_{0}$-semi-group $\Phi(t, s)=\exp A(t-s)$ generated by $A$. If moreover $B(\cdot) u(\cdot) \in L_{\mathrm{loc}}^{1}\left(\mathbf{R}_{+} ; X\right)$ then

$$
\begin{aligned}
& x(t)=\Phi(t, 0) x_{0}+\int_{0}^{t} \Phi(t, s) B(s) u(s) \mathrm{d} s, t \geq 0 \\
& x(\cdot) \in C\left(\mathbf{R}_{+} ; X\right) .
\end{aligned}
$$

Unfortunately a strong bang-bang-principle of the form (5) does not hold in infinite dimensions. However, there is a weaker version of it, see Papageorgiu [22]. In the infinite-dimensional case, instead of (4) we have

$$
{\overline{\mathcal{U}_{U}^{T}}}^{w}=\mathcal{U}_{\overline{\operatorname{conv}} U}^{T}
$$

where the $w$-closure refers to the weak topology in $L^{1}(0, T ; X)$. See Papageorgiu [22], Theorem 4.1. Moreover, if for all $t \geq 0$ the set $U(t)$ is weakly compact and convex, and $\mathcal{U}_{\operatorname{ext} U}^{T}$ is nonempty we have

$$
\overline{\mathcal{U}}_{\operatorname{ext} U}^{T}=\mathcal{U}_{U}^{T}
$$

(Th. 4.2 in [22]) and one obtains

$$
{\overline{\mathcal{R}_{\text {ext } U}^{T}}}^{w}\left(x_{0}\right)={\overline{\mathcal{R}_{U}^{T}}}^{w}\left(x_{0}\right) .
$$

Moreover, both sets are convex. Define $|U(t)|=\sup _{u(t) \in U(t)}\|u(t)\|$. If $|U(\cdot)| \in L_{\text {loc }}^{1}$ then $\mathcal{R}_{U}^{T}$ is already weakly closed and the corresponding closure can be omitted, that is

$$
{\overline{\mathcal{R}_{\text {ext } U}^{T}}}^{w}\left(x_{0}\right)=\mathcal{R}_{U}^{T}\left(x_{0}\right)
$$

See Papageorgiu [22] for further details. This result can be interpreted as an infinite dimensional anologon of the bang-bang principle (5), as it states that all reachable points can be approximated via a weakly-converging sequence of extremal controls in the sense of the weak topology in $L^{1}(0, T ; X)$. In terms of approximate controllability, equation (12) can also be interpreted as the statement that for the attainable set, weak approximate controllability by extremal controls holds. 
The theorem behind the bang-bang principle in the finite-dimensional case is the Liapunov [19] theorem on the convexity and compactness of vector valued measures. There are only few papers related to the extension of this result into infinite dimensions. See Papageorgiu [22], Drobot [5] (for convexity) and Dovretzki [4].

In other words, in infinite dimensions, under canonical circumstances, any reachable state can be approximated weakly by bang-bang controls.

For boundary control problems, of course, the input operator $B(t)$ is unbounded. In such a situation a corresponding general result does not seem to be known. This paper is meant to reveal further properties.

\subsubsection{Optimal control and bang-bang}

The bang-bang result (5) for the finite-dimensional case merely states that among possibly many other controls steering $x_{0}$ to $z$ in time $T>0$ there is a bang-bang control. In the infinite dimensional context (12) states that there is a sequence of extremal controls such that the sequence of generated states converges to $z$ in the sense of the weak topology in $L^{1}(0, T ; X)$ in time $T>0$.

In the framework of optimal control it is of interest whether optimal controls of bang-bang type exist. There are two interesting types of optimal control problems in this context: the minimal norm reachability problem, and the time optimal reachability problem. In the minimal norm problem one fixes a time $T$ and a reachable point $z$ and asks for a control $u$ that realizes the transfer from $x_{0}$ to $z$ with minimal norm $\|u\|$. In this case, $U(t)=X$.

Let $B_{\rho}(0)$ denotes the ball with radius $\rho$ in a given norm. In the time-optimal reachability problem one fixes the control set $U(t)=B_{\rho}(0)$ and asks for the minimal time $T>0$ when $z \in \mathcal{R}_{U}^{T}\left(x_{0}\right)$. For the latter problem, in any dimensions, it can be seen that the state $x(T)$ lies on the boundary of the reachable set that is

$$
x(T) \in \partial \mathcal{R}_{U}^{T}\left(x_{0}\right) .
$$

It is then a matter of applying separation theorems in order to characterize such boundary points. This question is answered by the classical Pontriagin-Maximum-Principle. In the linear finite-dimensional context of (2) we have

$$
\begin{aligned}
x(T) \in \partial \mathcal{R}_{U}^{T}\left(x_{0}\right) & \Leftrightarrow \exists \eta \text { such that } \\
\dot{\eta} & =A(t)^{\top} \eta \\
\eta(t)^{\top} B(t) u(t) & =\max _{u \in U} \eta(t)^{\top} B(t) u, \text { for almost all } t \in[0, T] .
\end{aligned}
$$

Here $\eta(t)$ is the adjoint state. The proof of this fact starts from a separating hyperplane, separating the boundary point $x(T)$ from the set $\mathcal{R}_{U}^{T}$. The normal to that hyperplane at $x(T)$ is considered as the end-point $\eta(T)$ of the adjoint problem in (13). The uniqueness of such controls steering an initial datum to a boundary point of the reachable set, therefore, is related to the geometry of that set. A suggestive notion in this context is that of normality, which says exactly that two controls reaching the same boundary point should coincide almost everywhere. Normality can be seen to be equivalent to the statement that no component of the adjoint state vanishes on a set of positive measure. Further analysis is easily available for the autonomous (still finite dimensional) control problem. In the autonomous case this is equivalent to

(1) The reachable set is strictly convex.

(2) The adjoint state has no component that vanishes on an interval of positive measure.

(3) The stronger Kalman condition $\operatorname{rank}\left[b_{i}, A b_{i}, \ldots, A^{n-1} b_{i}\right]=n, i=1, \ldots, m$ holds where the $b_{i}$ denote the columns of $B$.

In contrast to the stronger Kalman condition, the Kalman condition requires only $\operatorname{rank}\left[B, A B, \ldots, A^{n-1} B\right]=n$.

Under normality, the unique time-minimal control for a given norm bound on the control is the same as the norm-minimal control for the minimal time $T$. Thus under normality, in finite dimensions, norm-minimal controls are uniquely given as bang-bang controls. Such a result is not available in infinite dimensions.

There is no simple extension of statements (1)-(3) into infinite dimensional systems. For autonomous infinite dimensional systems Krabs [14] has shown that time minimal solutions are norm minimal on the minimal time 
interval. If the minimal-norm function is strictly decreasing (which is implied by normality in finite dimension), then the reverse statement is also true. Still in the infinite dimensional situation this does not imply a strong bang-bang principle. A unifying theory seems to be lacking. Indeed, the results concerning the wave equation and the heat equation differ substantially. Optimal solutions for the heat equation exhibit a weak bang-bang structure in the sense that the controls switch between the extreme points of the control constraint set, but the switches may accumulate at the time T. See Krabs [14], and Seidman [21]. Some results and open problems in the controllability of partial differential equations are presented in [28].

\subsection{Boundary control of the wave equation}

In this paper, we consider the problem of $L^{\infty}$-norm minimal Dirichlet boundary control of the wave equation, where at the left end the value zero is prescribed and the right end is controlled. An overview about control and numerical approximation of waves is given in [27].

We start with the problem of optimal exact control, where the control time and a desired terminal state that has to be reached at that time are given. We analyze the corresponding reachable set and show that in general, not even feasible bang-bang controls exist. In fact the geometry of the feasible set is not what one might expect: In the case of ordinary differential equations, the bang-bang controls are the extreme points of the feasible set (see [1]). In the case of the wave equation, the situation is completely different and the extreme points of the corresponding feasible set are not necessarily of bang-bang type. We show that for certain control times, the difference between the initial and the terminal state can only attain a finite number of values and the terminal velocities that can be reached by bang-bang controls with a finite number of switching points are equal to the initial velocity plus a linear combination of Dirac measures.

We introduce a primal problem for which the optimal control problem is the dual problem. Similar primal problems have been introduced in $[6,7,26]$. We show by an example, that in general, the primal problem does not have a solution. This motivates the introduction of a dual problem with measure-valued solutions for which existence of a solution is guaranteed. We present weak and strong duality results. In this context, weak duality means that the optimal value of the primal minimization problem is greater than or equal to the optimal value of the corresponding dual maximization problem and strong duality means that the optimal value of the primal problem is equal to the optimal value of the corresponding dual problem.

We continue with the problem of optimal approximate control, where the control time and an $\varepsilon$-neighbourhood of the desired terminal state that has to be reached at that time are given. The $\varepsilon$-neighbourhood is defined with the norms corresponding to the maximal regularity that we can expect in general, in our case this means with norms for non-reflexive spaces. With examples we show that also for the problem of optimal approximate control with relaxation in a non-reflexive space, it is necessary to consider measure-valued dual solutions. For these solutions, the corresponding weak form of a bang-bang principle is in general no restriction on the form of the controls: If the dual solutions are given by Dirac measures, the weak form of a bang-bang principle is only a restriction on a finite number of points on the time-interval.

For relaxation with respect to Hilbert-space norms, the situation is different: we give an example with an optimal control with bang-intervals and where the corresponding dual solution can be interpreted as a function.

This paper has the following structure: First we present the initial-boundary-value problem for the wave equation and provide a representation of its solution. Then we define bang-bang-off controls and compute the corresponding reachable set for certain times. A bang-bang-off control allows to use the value zero in addition to the two extremal control values. Such control strategies appear for example in orbital transfer and include the possibility of zero thrust, that is to switch off the rocket. For the wave equation, in [2] a time-optimal boundary control of bang-bang-off type is given. It turns out that the reachable set for the bang-bang-off controls that contains the reachable set for the bang-bang controls is very small.

Then we look at a problem of $L^{\infty}$-norm minimal control, where the feasible controls steer the system exactly to the zero state at a prescribed time. We consider an example for which a primal problem corresponding to this optimal control problem does not have a solution that is given as a function. 
This is the motivation to present a dual problem with solutions that are given as measures. We show weak and strong duality between this dual problem and our optimal control problem. The proof of strong duality shows how the sign of a measure that solves the dual problem is a constraint for the $L^{\infty}$-norm optimal controls that solve our original problem. The objective function of the dual problem contains an expression of $L^{1}$-norm type. The signum function is the subdifferential of the absolute value function that appears in this expression when we consider the necessary optimality conditions.

If the support of a certain measure that is related to a solution of the dual problem is equal to the whole time interval, an optimal bang-bang control exists. In the next part of the paper, we consider the problem of optimal $\varepsilon$-approximate control and introduce the corresponding dual problem. Weak and strong duality and the weak bang-bang principle (49) are proven. The result are analogous to the case of optimal exact control. The continuity of the optimal value function for the problem of optimal $\varepsilon$-approximate control as $\varepsilon$ tends to zero is shown. An example is given, where an optimal $\varepsilon$-approximate control can be computed explicitly which is not of bang-bang type. For this example, also solutions of the dual problem are given: In these dual solutions, Dirac measures appear.

As an alternative approach to the relaxation with a norm of $L^{\infty}$-type, we present a problem of optimal $\varepsilon$-approximate control where the relaxation of the end-condition is done with respect to a Hilbert-space norm. In contrast to the relaxation with a norm of $L^{\infty}$-type, the Hilbert space relaxation yields controls with bangintervals. As the relaxation parameter tends to zero, these intervals become shorter and shorter. This is illustrated by an example.

In the last section, we point out the relation between time-optimal and norm-minimal control (see also [15]) and give an example where the standard normality condition is violated: The minimal-norm function is piecewise constant and decreasing, but not strictly decreasing.

\section{The initial-Boundary-VAlue PROBlem AND ITS SOlUtion}

Let $L>0$ and a wave speed $c>0$ be given. For $y_{0} \in L^{\infty}(0, L), y_{1} \in W^{-1, \infty}(0, L)$ and a control function $u \in L^{\infty}(0, \infty)$ we consider the initial boundary value problem

$$
\begin{gathered}
\frac{1}{c^{2}} \frac{\partial^{2}}{\partial t^{2}} y(x, t)-\frac{\partial^{2}}{\partial x^{2}} y(x, t)=0,(x, t) \in(0, L) \times(0, \infty) \\
y(x, 0)=y_{0}(x), \frac{\partial}{\partial t} y(x, 0)=y_{1}(x), x \in(0, L) \\
y(0, t)=0, y(L, t)=u(t), t \in(0, \infty) .
\end{gathered}
$$

We give a representation of the solution of the initial-boundary-value problem that is based upon D'Alembert's solution of the wave equation. The given representation of the solution is very flexible with repect to the choice of function spaces containing $y_{0}$ and $y_{1}$.

Theorem 2.1. Let $t_{0}=L /$ c. For $(x, t) \in(0, L) \times(0, \infty)$ choose $i, j \in \mathbb{N}$ and $t_{1}, t_{2} \in\left[0, t_{0}\right)$ such that

$$
x+c t=j L+c t_{1},(L-x)+c t=i L+c t_{2} .
$$

Then the solution of the initial-boundary-value problem (15), (16), (17) is given by

$$
y(x, t)=\left[\alpha_{j}\left(t_{1}\right)+\beta_{i}\left(t_{2}\right)\right] / 2
$$

and the time derivative in the sense of distributions is given by

$$
\frac{\partial}{\partial t} y(x, t)=\frac{\alpha_{j}^{\prime}\left(t_{1}\right)+\beta_{i}^{\prime}\left(t_{2}\right)}{2}
$$


where for $s \in\left(0, t_{0}\right), \alpha_{k}$ and $\beta_{k}$ are defined as follows:

If $k \in \mathbb{N}$ is odd:

If $k \in \mathbb{N}$ is even:

$$
\begin{aligned}
& \alpha_{k}(s)=\sum_{i=0}^{(k-1) / 2} 2 u\left(s+2 i t_{0}\right)-\beta_{0}(s), \\
& \beta_{k}(s)=\sum_{i=1}^{(k-1) / 2}-2 u\left(s+(2 i-1) t_{0}\right)-\alpha_{0}(s) .
\end{aligned}
$$

$$
\begin{aligned}
& \alpha_{k}(s)=\sum_{i=0}^{(k-2) / 2} 2 u\left(s+(2 i+1) t_{0}\right)+\alpha_{0}(s), \\
& \beta_{k}(s)=\sum_{i=0}^{(k-2) / 2}-2 u\left(s+2 i t_{0}\right)+\beta_{0}(s) .
\end{aligned}
$$

For $s \in\left(0, t_{0}\right), \alpha_{0}$ and $\beta_{0}$ are defined as follows:

$$
\begin{aligned}
& \alpha_{0}(s)=y_{0}(c s)+\frac{1}{c} \int_{0}^{c s} y_{1}(\sigma) \mathrm{d} \sigma, \\
& \beta_{0}(s)=y_{0}(L-c s)-\frac{1}{c} \int_{0}^{L-c s} y_{1}(\sigma) \mathrm{d} \sigma .
\end{aligned}
$$

Proof. First we check the initial conditions: If $t=0$, we have $t_{1}=x / c$ and $t_{2}=(L-x) / c=t_{0}-t_{1}$ and $i=j=0$. Thus

Moreover, we have

$$
y(x, 0)=\left[\alpha_{0}\left(t_{1}\right)+\beta_{0}\left(t_{2}\right)\right] / 2=\left[y_{0}(x)+y_{0}(x)\right] / 2=y_{0}(x) .
$$

Hence the initial conditions (16) are valid.

$$
\left[\alpha_{0}^{\prime}\left(t_{1}\right)+\beta_{0}^{\prime}\left(t_{2}\right)\right] / 2=y_{1}(x) .
$$

Now we check whether the wave equation (15) holds. We have

$$
\begin{gathered}
\frac{1}{c^{2}} \frac{\partial^{2}}{\partial t^{2}} y(x, t)=\left[\alpha_{j}^{\prime \prime}\left(t_{1}\right)+\beta_{i}^{\prime \prime}\left(t_{2}\right)\right] /\left(2 c^{2}\right) \\
\frac{\partial^{2}}{\partial x^{2}} y(x, t)=\frac{1}{2} \frac{\partial^{2}}{\partial x^{2}}\left[\alpha_{j}\left(x / c+t-j t_{0}\right)+\beta_{i}\left(t_{0}-(x / c)+t-i t_{0}\right)\right]=\frac{\alpha_{j}^{\prime \prime}\left(t_{1}\right)+\beta_{i}^{\prime \prime}\left(t_{2}\right)}{2 c^{2}},
\end{gathered}
$$

hence (15) is valid.

Now we check the boundary condition $y(0, t)=0$. For $x=0$ we have

$$
x+c t=c t=j L+c t_{1},(L-x)+c t=c t+L=i L+c t_{2}
$$

hence $c t=(i-1) L+c t_{2}$. Thus $i=j+1$ and $t_{2}=t_{1}$. Hence $y(0, t)=\left[\alpha_{j}\left(t_{1}\right)+\beta_{j+1}\left(t_{1}\right)\right] / 2$.

If $j \in \mathbb{N}$ is odd, this yields

$$
y(0, t)=\sum_{i=0}^{(j-1) / 2} u\left(t_{1}+2 i t_{0}\right)-\frac{\beta_{0}\left(t_{1}\right)}{2}+\sum_{i=0}^{(j-1) / 2}-u\left(t_{1}+2 i t_{0}\right)+\frac{\beta_{0}\left(t_{1}\right)}{2}=0 .
$$

If $j \in \mathbb{N}$ is even, this yields

$$
y(0, t)=\sum_{i=0}^{(j-2) / 2} u\left(t_{1}+(2 i+1) t_{0}\right)+\frac{\alpha_{0}\left(t_{1}\right)}{2}+\sum_{i=1}^{j / 2}-u\left(t_{1}+(2 i-1) t_{0}\right)-\frac{\alpha_{0}\left(t_{1}\right)}{2}=0 .
$$


Hence $y(0, t)=0$ for all $t>0$.

Now we check the boundary condition $y(L, t)=u(t)$. For $x=L$ we have

$$
x+c t=L+c t=j L+c t_{1},(L-x)+c t=c t=i L+c t_{2}
$$

hence $c t=(j-1) L+c t_{1}$. Thus $i=j-1$ and $t_{2}=t_{1}$. Hence $y(L, t)=\left[\alpha_{j}\left(t_{1}\right)+\beta_{j-1}\left(t_{1}\right)\right] / 2$.

If $j \in \mathbb{N}$ is odd, this yields

$$
y(L, t)=\sum_{i=0}^{(j-1) / 2} u\left(t_{1}+2 i t_{0}\right)-\frac{\beta_{0}\left(t_{1}\right)}{2}+\sum_{i=0}^{(j-3) / 2}-u\left(t_{1}+2 i t_{0}\right)+\frac{\beta_{0}\left(t_{1}\right)}{2}=u\left(t_{1}+(j-1) t_{0}\right)=u(t) .
$$

If $j \in \mathbb{N}$ is even, this yields

$$
y(L, t)=\sum_{i=0}^{(j-2) / 2} u\left(t_{1}+(2 i+1) t_{0}\right)+\frac{\alpha_{0}\left(t_{1}\right)}{2}+\sum_{i=1}^{(j-2) / 2}-u\left(t_{1}+(2 i-1) t_{0}\right)-\frac{\alpha_{0}\left(t_{1}\right)}{2}=u\left(t_{1}+(j-1) t_{0}\right)=u(t) .
$$

Hence $y(L, t)=u(t)$ for all $t>0$ and the boundary conditions (17) hold.

\section{ReACHABle SET: Which STATES CAN BE REACHED BY BANG-BANG-OFF CONTROLS?}

In this section, we consider bang-bang-off (bang-zero-bang) controls where in addition to the two extremal control values, also zero is allowed as control value. The attainable set for bang-bang-off controls contains the set that can be reached using bang-bang controls. We show that the attainable set for bang-bang-off controls is rather small (see Th. 3.1).

Definition 3.1. Let $T>0$ be given. A function $u \in L^{\infty}(0, T)$ is called a bang-bang-off control, if

$$
u(t) \in\left\{\|u\|_{\infty,(0, T)}, 0,-\|u\|_{\infty,(0, T)}\right\}
$$

almost everywhere on $[0, T]$. A function $u \in L^{\infty}(0, T)$ is called a bang-bang control, if

$$
u(t) \in\left\{\|u\|_{\infty,(0, T)}, \quad-\|u\|_{\infty,(0, T)}\right\}
$$

almost everywhere on $[0, T]$.

Figure 2 shows an example for a bang-bang-off control. In [2] an example of a time-optimal bang-bang-off control of the wave equation with Neumann boundary controls at both ends is given.

Let $k \in \mathbb{N}$ and $T=2 k t_{0}$. We are interested in the set of states $y(x, T), y_{t}(x, T)$ that can be reached at the time $T$ with bang-bang-off controls.

Since $x+c T=x+2 k L$, we have $t_{1}=x / c$ and $j=2 k$. Since $(L-x)+c T=(L-x)+2 k L$, we have $t_{2}=(L-x) / c=t_{0}-t_{1}$ and $i=j$. This implies the equation

$$
\begin{aligned}
y(x, T) & =\left[\alpha_{2 k}\left(t_{1}\right)+\beta_{2 k}\left(t_{0}-t_{1}\right)\right] / 2 \\
& =\sum_{i=0}^{k-1} u\left(t_{1}+(2 i+1) t_{0}\right)+\sum_{i=0}^{k-1}-u\left(t_{0}-t_{1}+2 i t_{0}\right)+\left[\alpha_{0}\left(t_{1}\right)+\beta_{0}\left(t_{0}-t_{1}\right)\right] / 2 \\
& =y_{0}(x)+\sum_{i=0}^{k-1} u\left((2 i+1) t_{0}+t_{1}\right)-u\left((2 i+1) t_{0}-t_{1}\right) \\
& =y_{0}(x)+S_{1}(x / c) .
\end{aligned}
$$


If $u$ is a bang-bang-off control, the sum $S_{1}(x / c)$ can only obtain $4 k+1$ different values, namely from the set

$$
\left\{-2 k\|u\|_{\infty,(0, T)},(-2 k+1)\|u\|_{\infty,(0, T)}, \ldots,(2 k-1)\|u\|_{\infty,(0, T)}, 2 k\|u\|_{\infty,(0, T)}\right\} .
$$

Hence the set of states that can be reached with a bang-bang-off control $u$ is equal to

$$
\left\{f(x) \in L^{\infty}(0, L): \text { for all } x \in(0, L) \text { we have } f(x) \in y_{0}(x)+\|u\|_{\infty,(0, T)}\{-2 k,-2 k+1, \ldots, 2 k-1,2 k\}\right\} .
$$

This implies the following controllability result:

The state $y(x, T)=0$ can be reached at the time $T=2 k t_{0}$ if and only if the function $y_{0}$ attains at most $4 k+1$ different values from an equidistant grid of $4 k+1$ different values.

For the velocity at time $T$ we have

$$
\begin{aligned}
\frac{\partial}{\partial t} y(x, T) & =\frac{\alpha_{2 k}^{\prime}\left(t_{1}\right)+\beta_{2 k}^{\prime}\left(t_{0}-t_{1}\right)}{2} \\
& =\sum_{i=0}^{k-1} u^{\prime}\left((2 i+1) t_{0}+t_{1}\right)-u^{\prime}\left((2 i+1) t_{0}-t_{1}\right)+\left[\alpha_{0}^{\prime}\left(t_{1}\right)+\beta_{0}^{\prime}\left(t_{0}-t_{1}\right)\right] / 2 \\
& =y_{1}(x)+\sum_{i=0}^{k-1} u^{\prime}\left((2 i+1) t_{0}+t_{1}\right)-u^{\prime}\left((2 i+1) t_{0}-t_{1}\right) \\
& =y_{1}(x)+S_{2}(x / c) .
\end{aligned}
$$

For a bang-bang-off control with a finite number of switching points, we have

$$
u^{\prime}(t)=\sum_{j=0}^{N} \lambda_{j} \delta_{t_{j}}(t)
$$

with $t_{j} \in[0, T](j \in\{0, \ldots, N\})$ and $\lambda_{j} \in\left\{-2\|u\|_{\infty,(0, T)},-\|u\|_{\infty,(0, T)}, 0,\|u\|_{\infty,(0, T)}, 2\|u\|_{\infty,(0, T)}\right\}$.

In this case the sum $S_{2}(x / c)$ is a linear combination of Dirac-measures, that is

$$
S_{2}(x / c)=\sum_{i=0}^{k-1} \sum_{j=0}^{N} \lambda_{j}\left[\delta_{t_{j}}\left((2 i+1) t_{0}+x / c\right)-\delta_{t_{j}}\left((2 i+1) t_{0}-x / c\right)\right] .
$$

This implies the following controllability result: the velocity $y_{t}(x, T)$ can be reached in time $T=2 k t_{0}$ with a bang-bang-off control with a finite number of switching points, if and only if

$$
y_{t}(x, T)=y_{1}(x)+\sum_{i=0}^{k-1} \sum_{j=0}^{N} \lambda_{j}\left[\delta_{t_{j}}\left((2 i+1) t_{0}+x / c\right)-\delta_{t_{j}}\left((2 i+1) t_{0}-x / c\right)\right] .
$$

We summarize our controllability results in the following theorem:

Theorem 3.1. Let $T=2 k t_{0}$ and a bang-bang-off control $u \in L^{\infty}(0, T)$ be given. For the state that can be reached at time $T$ we have for $x \in(0, L)$ almost everywhere

$$
y(x, T) \in y_{0}(x)+\|u\|_{\infty,(0, T)}\{-2 k,-2 k+1, \ldots, 2 k-1,2 k\} .
$$




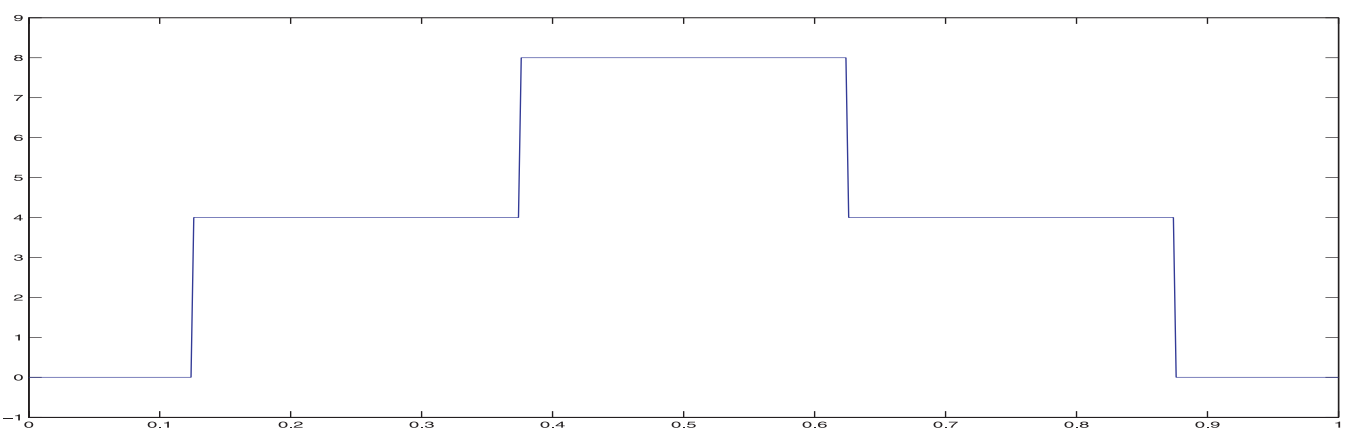

Figure 1. The state $y_{0}$ in Example 3.1.

For the velocity that can be reached at time $T$ with a bang-bang-off control with a finite number of switching points we have the representation

$$
y_{t}(x, T)=y_{1}(x)+\sum_{i=0}^{k-1} \sum_{j=0}^{N} \lambda_{j}\left[\delta_{t_{j}}\left((2 i+1) t_{0}+x / c\right)-\delta_{t_{j}}\left((2 i+1) t_{0}-x / c\right)\right]
$$

with $\lambda_{i} \in\left\{-2\|u\|_{\infty,(0, T)},-\|u\|_{\infty,(0, T)}, 0,\|u\|_{\infty,(0, T)}, 2\|u\|_{\infty,(0, T)}\right\}$ and $t_{i} \in(0, T)$.

Theorem 3.1 implies that the zero state $y(x, T)=y_{t}(x, T)=0$ can be reached at time $T=2 k t_{0}$ with a bangbang-off control with a finite number of switching points if and only if the initial velocity is a linear combination of Dirac measures and the initial state attains at most $4 k+1$ equidistant different values from a set of the form $h\{-2 k,-2 k+1, \ldots, 2 k-1,2 k\}$ with a stepsize $h>0$.

Example 3.1. Figure 1 shows a state $y_{0}$ on the space interval $[0, L]=[0,1]$ that can be controlled exactly to the zero state in the time $T=4 L / c=4(c=1)$ by a bang-bang-off control if $y_{1}(x)=0$ :

$$
y_{0}(x)=\left\{\begin{array}{rlr}
0 & \text { if } & x \in[0,1 / 8) \cup[7 / 8,1] \\
4 & \text { if } & x \in[1 / 8,3 / 8) \cup[5 / 8,7 / 8) \\
8 & \text { if } & x \in[3 / 8,5 / 8)
\end{array}\right.
$$

Figure 2 shows a bang-bang-off control $u$ on the time interval $[0, T]$ that steers the state from Figure 1 to the zero state. This is an exact control with minimal $L^{\infty}$-norm $\|u\|_{\infty,(0,4)}=2$ (see Sect. 4 ).

$$
u(t)=\left\{\begin{array}{rcr}
0 & \text { if } & t \in[0,1 / 8) \cup[7 / 8,9 / 8) \cup[15 / 8,19 / 8) \cup[21 / 8,27 / 8) \cup[29 / 8,4] \\
2 & \text { if } & t \in[1 / 8,7 / 8) \cup[19 / 8,21 / 8) \\
-2 & \text { if } & t \in[9 / 8,15 / 8) \cup[27 / 8,29 / 8)
\end{array}\right.
$$

Note that reflection at an axis in $T / 2=2$ and multiplication by -1 yields another exact bang-bang-off control with the same $L^{\infty}$-norm.

Another exact control with the same $L^{\infty}$-norm that is not bang-bang-off is given by the 2-periodic function $u_{1}$ depicted in Figure 3 with

$$
u_{1}(t)=\left\{\begin{array}{rrr}
0 & \text { if } & t \in[0,1 / 8) \cup[7 / 8,9 / 8) \cup[15 / 8,2) \\
1 & \text { if } & t \in[1 / 8,3 / 8) \cup[5 / 8,7 / 8) \\
2 & \text { if } & t \in[3 / 8,5 / 8) \\
-1 & \text { if } & t \in[9 / 8,11 / 8) \cup[13 / 8,15 / 8) \\
-2 & \text { if } & t \in[11 / 8,13 / 8)
\end{array}\right.
$$




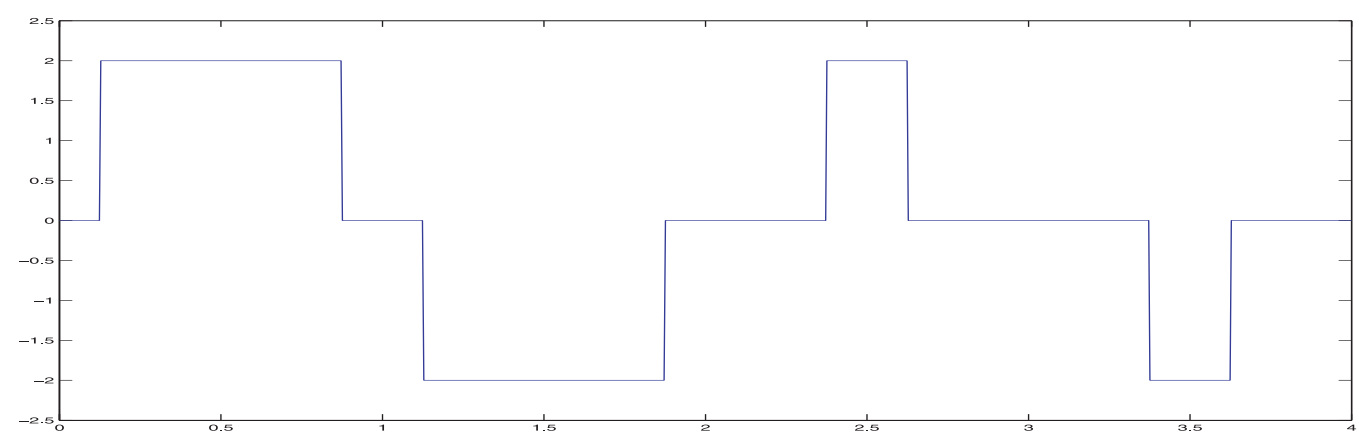

Figure 2. A successful bang-bang-off control in Example 3.1.

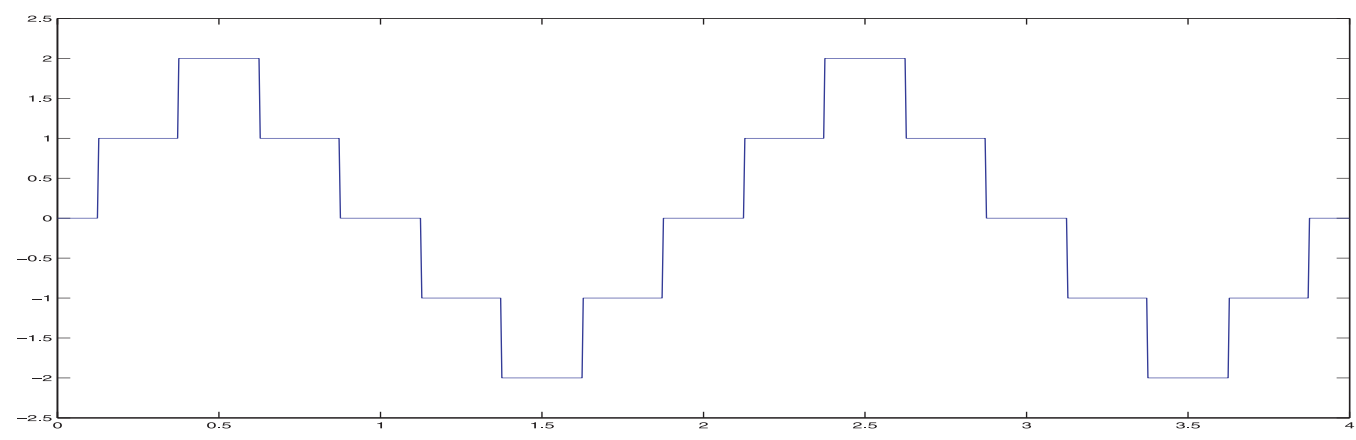

Figure 3. A successful control that is not bang-bang-off in Example 3.1.

\section{4. $L^{\infty}$-NORM MINIMAL EXACT CONTROL}

Consider the following problem of $L^{\infty}$-norm minimal control:

$$
P: \min (1 / 2)\|u\|_{\infty,(0, T)}^{2} \quad \text { such that } \quad(15)-(17) \quad \text { and } y(x, T)=y_{t}(x, T)=0 \text {. }
$$

Remark 4.1. Example 3.1 shows that in general, the solution of $P$ is not uniquely determined.

Exact controllability holds for $T \geq 2 t_{0}$ so if $T<2 t_{0}$, existence of a solution of $P$ cannot be guaranteed.

In [6], it is pointed out that the optimal boundary controls can also be determined as boundary traces of solutions of an adjoint optimal control problem (a primal problem). In this section we give an example, where the primal problem does not have a solution that is a Lebesgue-integrable function. However, the problem can be relaxed. In Section 5, we introduce a dual problem that has solutions in the sense of measures.

\subsection{An example with explicit solution}

Results from [10] imply that for $T=2 L / c, y_{0}(x)=\sin (x \pi / L)$ and $y_{1}(x)=0$ the control

$$
u(t)=\sin (c t \pi / L) / 2
$$


is a solution of the optimization problem $P$. Hence the optimal value of $P$ is equal to $1 / 8$. In fact, with $t_{1}=x / c$ Theorem 2.1 yields

$$
\begin{aligned}
y(x, T) & =u\left(t_{0}+t_{1}\right)-u\left(t_{0}-t_{1}\right)+y_{0}(x)=\sin \left(\frac{x}{L} \pi\right)+\frac{1}{2} \sin \left(\frac{c}{L}\left(t_{0}+t_{1}\right) \pi\right)-\frac{1}{2} \sin \left(\frac{c}{L}\left(t_{0}-t_{1}\right) \pi\right) \\
& =\sin \left(\frac{x}{L} \pi\right)+\frac{1}{2} \sin \left(\frac{c}{L} t_{1} \pi-\pi\right)+\frac{1}{2} \sin \left(\frac{c}{L} t_{1} \pi+\pi\right)=\sin \left(\frac{x}{L} \pi\right)+\sin \left(\frac{c}{L} t_{1} \pi\right) \cos (\pi)=0 .
\end{aligned}
$$

Moreover, we have

$$
\begin{aligned}
y_{t}(x, T) & =u^{\prime}\left(t_{0}+t_{1}\right)-u^{\prime}\left(t_{0}-t_{1}\right)+y_{1}(x)=\frac{c \pi}{2 L}\left[\cos \left(\frac{c}{L}\left(t_{0}+t_{1}\right) \pi\right)-\cos \left(\frac{c}{L}\left(t_{0}-t_{1}\right) \pi\right)\right] \\
& =-\frac{c \pi}{2 L}\left[\cos \left(\pi-\frac{c}{L} t_{1} \pi\right)-\cos \left(\pi+\frac{c}{L} t_{1} \pi\right)\right]=-\frac{c \pi}{L} \sin (\pi) \sin \left(\frac{c}{L} t_{1} \pi\right)=0 .
\end{aligned}
$$

The control $u$ is not bang-bang-off. In fact, Theorem 3.1 implies that no feasible bang-bang-off control exists.

The time $T=2 L / c$ is the minimal time for which the system is exactly controllable that is for which problem $P$ has feasible points for all initial states $y_{0}, y_{1}$ that are sufficiently regular. For $T=2 L / c$ all feasible controls $u_{1}$ have the form $u_{1}(t)=u(t)+r$ where $u$ is a given feasible control and $r$ is a real number. Hence the feasible set does not contain a bang-bang control.

\subsection{The corresponding primal problem}

Assume that $L=c=1$. For $\left(v_{0}, v_{1}\right) \in W^{1,1}(0,1) \times L^{1}(0,1)$ define

$$
K\left(\left(v_{0}, v_{1}\right)\right)=\frac{1}{2}\left(\int_{0}^{2}\left|v_{x}(1, t)\right| \mathrm{d} t\right)^{2}+\int_{0}^{1} y_{0}(x) v_{1}(x)-y_{1}(x) v_{0}(x) \mathrm{d} x
$$

where $v$ is the solution of the adjoint system

$$
\begin{aligned}
v_{t t}(x, t) & =v_{x x}(x, t) \\
v(0, t) & =v(1, t)=0 \\
v(x, 0) & =v_{0}(x), v_{t}(x, 0)=v_{1}(x) .
\end{aligned}
$$

The primal problem corresponding to problem $P$ is

$$
\text { PRIM : } \min K\left(\left(v_{0}, v_{1}\right)\right) .
$$

If $v$ is a solution of PRIM, then a solution $u$ of $P$ is quasi-bang-bang in the sense that

$$
u(t) \in \operatorname{sign}\left(v_{x}(1, t)\right) .
$$

Note that it is not clear that a solution $v$ of $P R I M$ exists in the given space $W^{1,1}(0,1) \times L^{1}(0,1)$. This will be explained further in Section 4.3.

Example 4.1. Consider problem $P$ with $L=c=1, T=4, y_{1}=0$ and $y_{0}$ as in (22). Then a solution of $P R I M$ is given by $v_{0}=0$ and

$$
v_{1}(x)=\left\{\begin{array}{rll}
0 & \text { if } & x \in[3 / 8,5 / 8), \\
-2 & \text { if } & x \notin[3 / 8,5 / 8) .
\end{array}\right.
$$

This follows from the strong duality results in Section 5. All the optimal controls presented in Example 3.1 are quasi-bang-bang in the sense of (27). This is illustrated by Figure 4 that shows the state $v$ generated by $\left(v_{0}, v_{1}\right)$ as defined above. The support of $v_{x}(1, \cdot)$ is equal to $[3 / 8,5 / 8] \cup[11 / 8,13 / 8] \cup[19 / 8,21 / 8] \cup[27 / 8,29 / 8]$. 


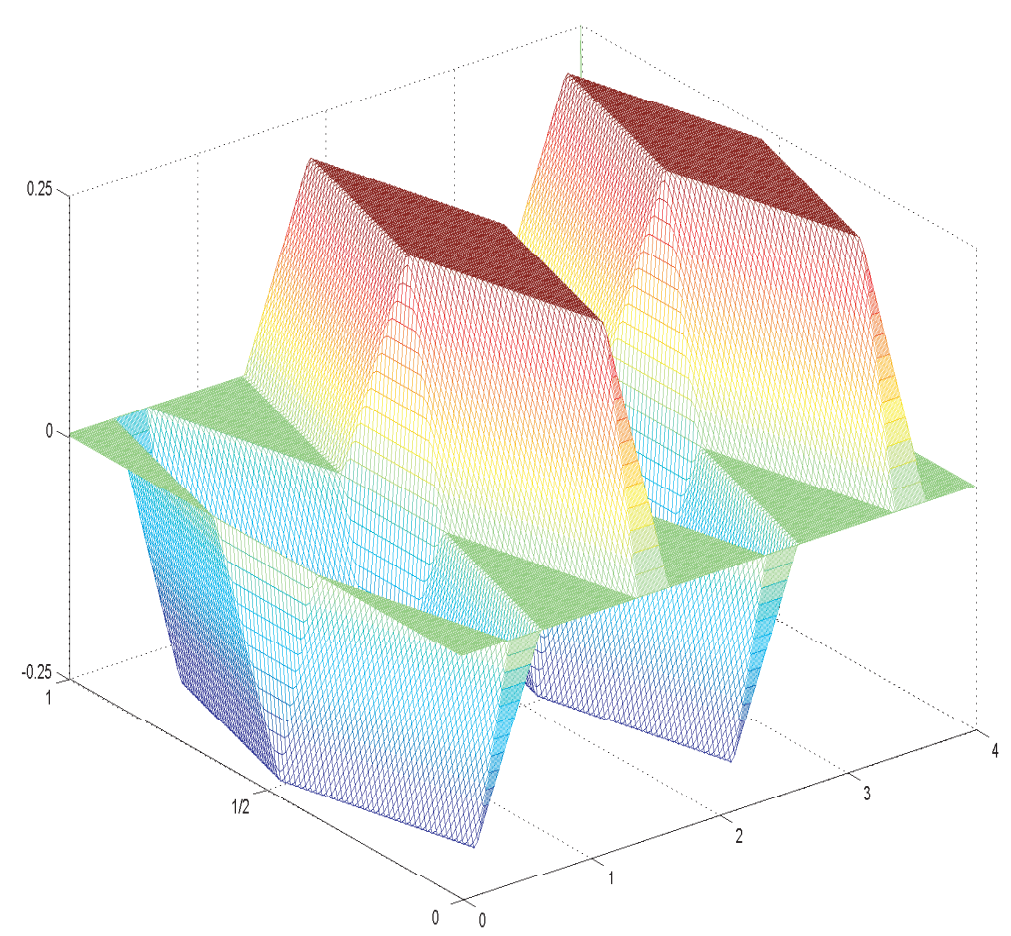

FigurE 4. The optimal adjoint state in Example 4.1.

\subsection{Example: nonexistence of a solution of the primal problem}

In this section, for our example with $y_{0}(x)=\sin (x \pi / L)$ and $y_{1}(x)=0$ we construct a minimizing sequence that has a Dirac measure as the only limit point. We show that it makes sense to consider this Dirac measure as a solution of the primal problem PRIM.

For real numbers $M \in \mathbf{R}$ and $\delta<1 / 2$ define

$$
v_{0}(x)=0, v_{1}(x)=-M \text { for } x \in[(1 / 2)-\delta,(1 / 2)+\delta], v_{1}(x)=0 \text { else. }
$$

For $t \in\left(0, t_{0}\right)$ we have

$$
v_{x}(1, t)=\left[\alpha_{1}^{\prime}(t)-\beta_{0}^{\prime}(t)\right] / 2=-\beta_{0}^{\prime}(t)
$$

and for $t \in\left(t_{0}, 2 t_{0}\right)$ we have

$$
v_{x}(1, t)=\left[\alpha_{2}^{\prime}\left(t-t_{0}\right)-\beta_{1}^{\prime}\left(t-t_{0}\right)\right] / 2=\alpha_{0}^{\prime}\left(t-t_{0}\right) .
$$

This implies

$$
\int_{0}^{2}\left|v_{x}(1, t)\right| \mathrm{d} t=\int_{0}^{1}\left|\beta_{0}^{\prime}(t)\right| \mathrm{d} t+\int_{1}^{2}\left|\alpha_{0}^{\prime}\left(t-t_{0}\right)\right| \mathrm{d} t=\int_{0}^{1}\left|\alpha_{0}^{\prime}(t)\right|+\left|\beta_{0}^{\prime}(t)\right| \mathrm{d} t=2 \int_{0}^{1}\left|v_{1}(t)\right| \mathrm{d} t=4 \delta|M|
$$


Moreover, we have

$$
\begin{aligned}
\int_{0}^{1} y_{0}(x) v_{1}(x)-y_{1}(x) v_{0}(x) \mathrm{d} x & =\int_{0}^{1} \sin (\pi x) \frac{1}{2}\left[\alpha_{0}^{\prime}(x)+\beta_{0}^{\prime}(1-x)\right] \mathrm{d} x=-M \int_{(1 / 2)-\delta}^{(1 / 2)+\delta} \sin (\pi x) \mathrm{d} x \\
& =-\frac{2 M}{\pi}\left[\frac{1}{2}\left(\cos \left(\frac{\pi}{2}-\pi \delta\right)-\cos \left(\frac{\pi}{2}+\pi \delta\right)\right)\right]=-\frac{2 M}{\pi} \sin (\pi \delta)
\end{aligned}
$$

Hence

$$
K\left(\left(v_{0}, v_{1}\right)\right)=8 \delta^{2} M^{2}-\frac{2 M}{\pi} \sin (\pi \delta) .
$$

With the choice $\delta=1 /(8 M)$ we obtain

$$
K\left(\left(v_{0}, v_{1}\right)\right)=\frac{1}{8}-\frac{2 M}{\pi} \sin (\pi /(8 M)) \rightarrow-\frac{1}{8}(M \rightarrow \infty) .
$$

Define

$$
I_{1}=\int_{0}^{1}\left|\beta_{0}^{\prime}(t)\right| \mathrm{d} t+\int_{1}^{2}\left|\alpha_{0}^{\prime}\left(t-t_{0}\right)\right| \mathrm{d} t=\int_{0}^{1}\left|\alpha_{0}^{\prime}(t)\right|+\left|\beta_{0}^{\prime}(t)\right| \mathrm{d} t .
$$

Since $|\sin (\pi x)| \leq 1$, we have a lower bound for $K\left(\left(v_{0}, v_{1}\right)\right)$, namely

$$
K\left(\left(v_{0}, v_{1}\right)\right)=\frac{I_{1}^{2}}{2}+\int_{0}^{1} \sin (\pi x) \frac{1}{2}\left[\alpha_{0}^{\prime}(x)+\beta_{0}^{\prime}(1-x)\right] \mathrm{d} x \geq \frac{I_{1}^{2}}{2}-\frac{1}{2} \int_{0}^{1}\left|\alpha_{0}^{\prime}(t)\right|+\left|\beta_{0}^{\prime}(t)\right| \mathrm{d} t=\frac{1}{2}\left(I_{1}^{2}-I_{1}\right) \geq-\frac{1}{8} .
$$

This implies that the control functions $\left(v_{1, k}, v_{2, k}\right)$ as defined in (29) with the choice $M=M_{k}=k, \delta=\delta_{k}=$ $1 /(8 k)$ form a minimizing sequence. This in turn implies that the optimal value of problem $P R I M$ is $-1 / 8$.

Our minimizing sequence is a bounded sequence in $L^{1}$. For all $\varphi \in C(0,1)$, we have

$$
\lim _{k \rightarrow \infty} \int_{0}^{1} v_{1, k}(x) \varphi(x) \mathrm{d} x=\lim _{k \rightarrow \infty}-k \int_{(1 / 2)-\delta_{k}}^{(1 / 2)+\delta_{k}} \varphi(x) \mathrm{d} x=-(1 / 4) \varphi(1 / 2)
$$

which shows that the sequence $\left(v_{1, k}\right)_{k \in \mathbb{N}}$ does not have a limit point in $L^{1}(0,1)$.

We can even state that for our example, problem PRIM does not have a solution.

This can be seen as follows: Inequality $(30)$ show that $K(\cdot)$ can only obtain the value $-1 / 8$ if

$$
\int_{0}^{1} \sin (\pi x)\left[\alpha_{0}^{\prime}(x)+\beta_{0}^{\prime}(x)\right] \mathrm{d} x=-1 / 2 \text { and } I_{1}=\int_{0}^{1}\left|\alpha_{0}^{\prime}(x)\right|+\left|\beta_{0}^{\prime}(x)\right| \mathrm{d} x=1 / 2 .
$$

Since these two equations cannot be satisfied at the same time, problem PRIM cannot have a solution that is given as a function.

Instead, a solution given as a measure that describes the limit point of the minimizing sequence should be considered. On account of (31), for our example $v_{1}$ would be a Dirac measure with support in the point $x=1 / 2$ and weight $-1 / 4$, that is

With $v_{0}=0$ this yields

$$
v_{1}(t)=-(1 / 4) \delta_{1 / 2}(t)
$$

$$
\begin{array}{rll}
v_{x}(1, t)=(1 / 4) \delta_{1 / 2}(t) & \text { for } & t \in\left(0, t_{0}\right] \\
v_{x}(1, t)=-(1 / 4) \delta_{1 / 2}\left(t-t_{0}\right) & \text { for } & t \in\left(t_{0}, 2 t_{0}\right) .
\end{array}
$$

Hence in this case the quasi-bang-bang principle $u(t) \in \operatorname{sign}\left(v_{x}(1, t)\right)$ yields

$$
u(1 / 2)=\|u\|_{\infty,(0,2)} \text { and } u(3 / 2)=-\|u\|_{\infty,(0,2)}
$$


which is satisfied by the optimal control $u$ defined in (25).

\section{A DUAL PROBLEM}

In order to avoid the difficulties connected with problem PRIM, in this section we introduce a dual problem for $P$ in the spirit of the Hilbert uniqueness method (HUM) introduced by Lions (see [18]).

Define the topological dual spaces $X_{0}=\left(L^{\infty}(0, L)\right)^{*}$ and $X_{1}=\left(W^{-1, \infty}(0, L)\right)^{*}$. The dual of $L^{\infty}$ is larger than $L^{1}$ and may be identified with a space of absolutely continuous, finitely additive set functions of bounded total variation on $(0, L)$ (see [25], p. 118). For $\left(\mu_{0}, \mu_{1}\right) \in X_{1} \times X_{0}$, consider the adjoint system

$$
\begin{aligned}
& \mu_{t t}=c^{2} \mu_{x x}(x, t) \\
& \mu(x, 0)=\mu_{0}(x), \mu_{t}(x, 0)=\mu_{1}(x) \\
& \mu(0, t)=\mu(L, t)=0 .
\end{aligned}
$$

For $\left(\mu_{0}, \mu_{1}\right) \in X_{1} \times X_{0}$ define

$$
J\left(\left(\mu_{0}, \mu_{1}\right)\right)=-\frac{c^{4}}{2}\left(\int_{0}^{T} \mathrm{~d}\left|\mu_{x}(L, t)\right|\right)^{2}-\left\langle y_{0}, \mu_{1}\right\rangle_{L^{\infty} \times X_{0}}+\left\langle y_{1}, \mu_{0}\right\rangle_{W^{-1, \infty} \times X_{1}}
$$

where $\mu$ is the solution of the adjoint system (32)-(34). We define the dual problem

$$
D U A L: \max _{\left(\mu_{0}, \mu_{1}\right) \in X_{1} \times X_{0}} J\left(\left(\mu_{0}, \mu_{1}\right)\right) .
$$

Weak duality. In this section, we show that for all controls $u \in L^{\infty}(0, T)$ for which the solution $y$ of (15)-(17) satisfies the end conditions $y(x, T)=y_{t}(x, T)=0$ and for all $\left(\mu_{0}, \mu_{1}\right) \in X_{1} \times X_{0}$ we have

$$
J\left(\left(\mu_{0}, \mu_{1}\right)\right) \leq(1 / 2)\|u\|_{\infty,(0, T)}^{2}
$$

which shows that problems $P$ and $D U A L$ are in weak duality that is the optimal value of the minimization problem $P$ is greater than or equal to the optimal value of the maximization problem $D U A L$.

To show that weak duality holds, we multiply the state equation (15) (with the control $u$ ) by the solution $\mu$ of the adjoint system. Using the end conditions, this yields the equation

$$
c^{2} \int_{0}^{T} u(t) \mathrm{d} \mu_{x}(L, t)+\left\langle y_{0}, \mu_{1}\right\rangle_{L^{\infty} \times X_{0}}-\left\langle y_{1}, \mu_{0}\right\rangle_{W^{-1, \infty} \times X_{1}}=0 .
$$

Thus we have

$$
\begin{aligned}
\frac{1}{2}\|u\|_{\infty,(0, T)}^{2}-J\left(\left(\mu_{0}, \mu_{1}\right)\right) & =\frac{1}{2}\|u\|_{\infty,(0, T)}^{2}+\frac{1}{2}\left(c^{2} \int_{0}^{T} \mathrm{~d}\left|\mu_{x}(L, t)\right|\right)^{2}+\left\langle y_{0}, \mu_{1}\right\rangle_{L \infty \times X_{0}}-\left\langle y_{1}, \mu_{0}\right\rangle_{W^{-1, \infty} \times X_{1}} \\
& =\frac{1}{2}\|u\|_{\infty,(0, T)}^{2}-c^{2} \int_{0}^{T} u(t) \mathrm{d} \mu_{x}(L, t)+\frac{1}{2}\left(c^{2} \int_{0}^{T} \mathrm{~d}\left|\mu_{x}(L, t)\right|\right)^{2} \\
& \geq \frac{1}{2}\left[\|u\|_{\infty,(0, T)}^{2}-2 c^{2}\|u\|_{\infty,(0, T)} \int_{0}^{T} \mathrm{~d}\left|\mu_{x}(L, t)\right|+\left(c^{2} \int_{0}^{T} \mathrm{~d}\left|\mu_{x}(L, t)\right|\right)^{2}\right] \\
& =\frac{1}{2}\left[\|u\|_{\infty,(0, T)}-c^{2} \int_{0}^{T} \mathrm{~d} \mid \mu_{x}(L, t)\right]^{2} \geq 0 .
\end{aligned}
$$


So we have shown that the optimization problems $P$ and $D U A L$ are in weak duality.

Strong duality and detection of bang-bang controls. In this section, we show that if $D U A L$ has a solution, the optimal value of $D U A L$ is equal to the optimal value of $P$. Moreover, we show how a solution of $D U A L$ can be used to construct a solution of $P$.

For $x \in \mathbb{R}$ define $f(x)=|x|$ as the absolute value function. Then $f$ is a convex function. For the subdifferential (see [24]) of $f$, we use the notation $\partial f(x)=\operatorname{sign}(x)$.

Let $\left(\mu^{0}, \mu^{1}\right)$ be a solution of $D U A L$. We want to look at the necessary optimality conditions. Since the $L^{1}$ norm is not differentiable, we cannot look at the gradient of $-J$. However, for the subdifferential $\partial(-J)$ (see [3]) of the convex functional $-J(\cdot)$ we have

$$
0 \in \partial\left(-J\left(\left(\mu_{0}, \mu_{1}\right)\right)\right)
$$

This implies the existence of a function $w_{1} \in L^{\infty}(0, T)$ such that

$$
w_{1}(t) \in \operatorname{sign}\left(\mu_{x}(L, t)\right) \text { on }[0, T]
$$

and for all $\left(\omega_{0}, \omega_{1}\right) \in X_{1} \times X_{0}$ we have

$$
0=c^{4}\left(\int_{0}^{T} \mathrm{~d}\left|\mu_{x}(L, t)\right|\right) \int_{0}^{T} w_{1}(t) \mathrm{d} \omega_{x}(L, t)+\left\langle y_{0}, \omega_{1}\right\rangle_{L^{\infty} \times X_{0}}-\left\langle y_{1}, \omega_{0}\right\rangle_{W^{-1, \infty} \times X_{1}}
$$

where $\omega$ denotes the solution of the adjoint system (32)-(34) with the initial data $\left(\omega_{0}, \omega_{1}\right)$. Define

$$
I_{1}=\int_{0}^{T} \mathrm{~d}\left|\mu_{x}(L, t)\right|
$$

Equation (38) implies the existence of a control $u=c^{2} I_{1} w_{1} \in L^{\infty}(0, T)$ that satisfies the inclusion

$$
u(t) \in c^{2} I_{1} \operatorname{sign}\left(\mu_{x}(L, t)\right)
$$

and for all $\left(\omega_{0}, \omega_{1}\right) \in X_{1} \times X_{0}$ the equation

$$
0=c^{2} \int_{0}^{T} u(t) \mathrm{d} \omega_{x}(L, t)+\left\langle y_{0}, \omega_{1}\right\rangle_{L^{\infty} \times X_{0}}-\left\langle y_{1}, \omega_{0}\right\rangle_{W^{-1, \infty} \times X_{1}}
$$

Multiplying the state equation (15) (with the control $u$ ) by the solution $\omega$ of the adjoint system yields

$$
\begin{aligned}
0= & c^{2} \int_{0}^{T} u(t) \mathrm{d} \omega_{x}(L, t)+\left\langle y_{0}, \omega_{1}\right\rangle_{L^{\infty} \times X_{0}}-\left\langle y_{1}, \omega_{0}\right\rangle_{W^{-1, \infty} \times X_{1}} \\
& -\left\langle y(\cdot, T), \omega_{t}(\cdot, T)\right\rangle_{L^{\infty} \times X_{0}}+\left\langle y_{t}(\cdot, T), \omega(\cdot, T)\right\rangle_{W^{-1, \infty} \times X_{1}}
\end{aligned}
$$

Combining these two identities (41), (42) yields

$$
0=\left\langle y(\cdot, T), \omega_{t}(\cdot, T)\right\rangle_{L^{\infty} \times X_{0}}-\left\langle y_{t}(\cdot, T), \omega(\cdot, T)\right\rangle_{W^{-1, \infty} \times X_{1}}
$$

for all $\left(\omega_{0}, \omega_{1}\right) \in X_{1} \times X_{0}$, which is equivalent to the exact controllability condition.

This means that the control $u$ which satisfies (40) and (41) is feasible for $P$. As in (37), we see that

$$
\begin{aligned}
\frac{1}{2}\|u\|_{\infty,(0, T)}^{2}-J\left(\left(\mu_{0}, \mu_{1}\right)\right) & =\frac{c^{4}}{2} I_{1}^{2}-c^{4} \int_{0}^{T} I_{1} \operatorname{sign}\left(\mu_{x}(L, t)\right) \mathrm{d} \mu_{x}(L, t)+\frac{c^{4}}{2} I_{1}^{2} \\
& =c^{4} I_{1}^{2}-c^{4} I_{1} \int_{0}^{T} \mathrm{~d}\left|\mu_{x}(L, t)\right|=0
\end{aligned}
$$


This means that $P$ and $D U A L$ have the same optimal values and that the control $u$ that satisfies (40) and (41) solves $P$. Thus we have constructed an $L^{\infty}$ norm minimal control.

Note that (40) implies that

$$
u(t) \in\left\{-\|u\|_{\infty,(0, T)},\|u\|_{\infty,(0, T)}\right\} \text { for all } t \in \operatorname{supp}\left(\mu_{x}(L, \cdot)\right) .
$$

Since the set $\operatorname{supp}\left(\mu_{x}(L, \cdot)\right.$ can be very small (for example, it may contain only two points as in our previous example) this is not a restriction on the form of the control.

Suppose that $D U A L$ has a solution $\mu$ such that $\operatorname{supp}\left(\mu_{x}(L, \cdot)\right)=[0, T]$. Then the $L^{\infty}$ norm minimal control that satisfies (40) and (41) only attains the values $c^{2} I_{1}=\|u\|_{\infty,(0, T)}$ and $-c^{2} I_{1}$, that is $u$ is a bang-bang control.

Existence of a solution for the dual problem. In this section we show that problem $D U A L$ has a solution. On account of the weak duality, the objective functional $J$ is bounded above. Using the same technique as for the proof of Theorem 2.1, we can show that for all natural numbers $j$ and $t_{1} \in\left[0, t_{0}\right)$ we have

$$
\mu_{x}\left(L, t+(j-1) t_{0}+t_{1}\right)= \begin{cases}\mu_{0}^{\prime}(c s)+\mu_{1}(c s) / c & \text { if } j \text { is even, } \\ \mu_{0}^{\prime}(L-c s)-\mu_{1}(L-c s) / c & \text { if } j \text { is odd. }\end{cases}
$$

For $T \geq 2 t_{0}$ this implies the inequality

$$
I_{1}=\int_{0}^{T} \mathrm{~d}\left|\mu_{x}(L, t)\right| \geq \int_{0}^{t_{0}} \mathrm{~d}\left|\mu_{0}^{\prime}(c s)\right|+\frac{1}{c} \int_{0}^{t_{0}} \mathrm{~d}\left|\mu_{1}(c s)\right| .
$$

From the definition (35) of $J$, this implies the coerciveness of $-J$ on the space $X_{1} \times X_{0}$.

Thus every minimizing sequence for $-J$ is bounded in the space $X_{1} \times X_{0}$, which is the dual space of $W^{-1, \infty}(0, L) \times L^{\infty}(0, L)$. By Alaoglu's Theorem (see [23], Th. 2.5.2) this implies that every minimizing sequence contains a weakly* convergent subsequence. Inserting the representation of $\mu_{x}(L, t)$ from $(43)$ in the definition (35) of $J$ implies that $-J$ is sequentially weak* lower semicontinuous. Thus every limit point of the weakly* convergent subsequence solves $D U A L$.

Note that on account of the use of weak* convergence, this argument does not work for problem $P R I M$, since the space $W^{1,1}(0,1) \times L^{1}(0,1)$ containing the solutions of $P R I M$ is not given as the dual space of another Banach space.

\section{Optimal approximate CONTRol}

Now we analyze what happens when we replace the exact end condition

$$
y(\cdot, T)=y_{t}(\cdot, T)=0
$$

by the constraints

$$
\|y(\cdot, T)\|_{\infty,(0, L)} \leq \varepsilon \text { and }\left\|y_{t}(\cdot, T)\right\|_{W^{-1, \infty},(0, L)} \leq \varepsilon
$$

for some $\varepsilon>0$.

\subsection{The $\boldsymbol{L}^{\infty}$-norm minimal $\varepsilon$-approximate control problem $\boldsymbol{P}(\varepsilon)$}

Let $\varepsilon \geq 0$ be given.

Consider the following problem of $L^{\infty}$-norm minimal control:

$$
P(\varepsilon): \min (1 / 2)\|u\|_{\infty,(0, T)}^{2} \text { such that }(15)-(17) \text { and }\|y(\cdot, T)\|_{\infty,(0, L)} \leq \varepsilon \text { and }\left\|y_{t}(\cdot, T)\right\|_{W^{-1, \infty},(0, L)} \leq \varepsilon
$$

Problem $P(0)$ is equivalent to the problem $P$ of $L^{\infty}$-norm minimal exact control defined above. 


\subsection{A dual problem for $\varepsilon$-approximate control}

Let the functional $J$ be defined as in (35). For $\varepsilon \geq 0$, we define the dual problem

$$
D U A L(\varepsilon): \max _{\left(\mu_{0}, \mu_{1}\right) \in X_{1} \times X_{0}} J\left(\left(\mu_{0}, \mu_{1}\right)\right)-\varepsilon\left(\|\mu(\cdot, T)\|_{X_{1}}+\left\|\mu_{t}(\cdot, T)\right\|_{X_{0}}\right) .
$$

where $\mu$ is the solution of the adjoint system (32)-(34).

Problem $D U A L(0)$ is the dual problem defined in Section 5.

Weak duality for $\varepsilon$-approximate control. In this section, we show that for all controls $u \in L^{\infty}(0, T)$ for which the solution $y$ of (15)-(17) satisfies the $\varepsilon$-approximate end conditions (44) and for all $\left(\mu_{0}, \mu_{1}\right) \in X_{1} \times X_{0}$ we have

$$
J\left(\left(\mu_{0}, \mu_{1}\right)\right)-\varepsilon\left(\|\mu(\cdot, T)\|_{X_{1}}+\left\|\mu_{t}(\cdot, T)\right\|_{X_{0}}\right) \leq(1 / 2)\|u\|_{\infty,(0, T)}^{2}
$$

which shows that problems $P(\varepsilon)$ and $D U A L(\varepsilon)$ are in weak duality. This implies in particular that the optimal value of $D U A L(\varepsilon)$ is less than or equal to the optimal value of $P(\varepsilon)$.

To show that weak duality holds, we multiply the state equation (15) (with the control $u$ ) by the solution $\mu$ of the adjoint system. This yields the equation

$$
\begin{aligned}
0= & c^{2} \int_{0}^{T} u(t) \mathrm{d} \mu_{x}(L, t)+\left\langle y_{0}, \mu_{1}\right\rangle_{L^{\infty} \times X_{0}}-\left\langle y_{1}, \mu_{0}\right\rangle_{W-1, \infty \times X_{1}} \\
& -\left\langle y(\cdot, T), \mu_{t}(\cdot, T)\right\rangle_{L^{\infty} \times X_{0}}+\left\langle y_{t}(\cdot, T), \mu(\cdot, T)\right\rangle_{W^{-1, \infty} \times X_{1}} .
\end{aligned}
$$

Using the $\varepsilon$-approximate end conditions, this implies the inequality

$$
c^{2} \int_{0}^{T} u(t) \mathrm{d} \mu_{x}(L, t)+\left\langle y_{0}, \mu_{1}\right\rangle_{L^{\infty} \times X_{0}}-\left\langle y_{1}, \mu_{0}\right\rangle_{W^{-1, \infty} \times X_{1}} \geq-\varepsilon\left(\|\mu(\cdot, T)\|_{X_{1}}+\left\|\mu_{t}(\cdot, T)\right\|_{X_{0}}\right) .
$$

Thus we have

$$
\begin{aligned}
\frac{1}{2}\|u\|_{\infty,(0, T)}^{2}- & J\left(\left(\mu_{0}, \mu_{1}\right)\right)+\varepsilon\left(\|\mu(\cdot, T)\|_{X_{1}}+\left\|\mu_{t}(\cdot, T)\right\|_{X_{0}}\right) \\
= & \frac{1}{2}\|u\|_{\infty,(0, T)}^{2}+\frac{c^{4}}{2}\left(\int_{0}^{T} \mathrm{~d}\left|\mu_{x}(L, t)\right|\right)^{2}+\varepsilon\left(\|\mu(\cdot, T)\|_{X_{1}}+\left\|\mu_{t}(\cdot, T)\right\|_{X_{0}}\right) \\
& +\left\langle y_{0}, \mu_{1}\right\rangle_{L^{\infty} \times X_{0}}-\left\langle y_{1}, \mu_{0}\right\rangle_{W}-1, \infty \times X_{1} \\
\geq & \frac{1}{2}\|u\|_{\infty,(0, T)}^{2}-c^{2} \int_{0}^{T} u(t) \mathrm{d} \mu_{x}(L, t)+\frac{1}{2}\left(c^{2} \int_{0}^{T} \mathrm{~d}\left|\mu_{x}(L, t)\right|\right)^{2} \\
\geq & \frac{1}{2}\left[\|u\|_{\infty,(0, T)}^{2}-2 c^{2}\|u\|_{\infty,(0, T)} \int_{0}^{T} \mathrm{~d}\left|\mu_{x}(L, t)\right|+\left(c^{2} \int_{0}^{T} \mathrm{~d}\left|\mu_{x}(L, t)\right|\right)^{2}\right] \\
= & \frac{1}{2}\left[\|u\|_{\infty,(0, T)}-c^{2} \int_{0}^{T} \mathrm{~d}\left|\mu_{x}(L, t)\right|\right]^{2} \geq 0 .
\end{aligned}
$$

So we have shown that the optimization problems $P(\varepsilon)$ and $D U A L(\varepsilon)$ are in weak duality.

Strong duality for $\varepsilon$-approximate control. In this section, we show that if $D U A L(\varepsilon)$ has a solution, the optimal value of $D U A L(\varepsilon)$ is equal to the optimal value of $P(\varepsilon)$ and show how a solution of $D U A L(\varepsilon)$ can be used to construct a solution of $P(\varepsilon)$. 
Let $\left(\mu^{0}, \mu^{1}\right)$ be a solution of $D U A L(\varepsilon)$. We want to look at the necessary optimality conditions. Since the absolute value function is not differentiable, we cannot look at the gradient of the objective functional

$$
J-\varepsilon\left(\|\mu(\cdot, T)\|_{X_{1}}+\left\|\mu_{t}(\cdot, T)\right\|_{X_{0}}\right)
$$

For the subdifferential $\partial\left(-J+\varepsilon\left(\|\mu(\cdot, T)\|_{X_{1}}+\left\|\mu_{t}(\cdot, T)\right\|_{X_{0}}\right)\right.$ (see [3]) of the corresponding convex functional we have

$$
0 \in \partial\left(\varepsilon\left(\|\mu(\cdot, T)\|_{X_{1}}+\left\|\mu_{t}(\cdot, T)\right\|_{X_{0}}\right)-J\left(\left(\mu_{0}, \mu_{1}\right)\right)\right) .
$$

This implies the existence of a function $w_{1} \in L^{\infty}(0, T)$ such that

$$
w_{1}(t) \in \operatorname{sign}\left(\mu_{x}(L, t)\right) \text { on }[0, T]
$$

and for all $\left(\omega_{0}, \omega_{1}\right) \in X_{1} \times X_{0}$ we have

$$
\begin{gathered}
0=c^{4} \int_{0}^{T} \mathrm{~d}\left|\mu_{x}(L, t)\right| \int_{0}^{T} w_{1}(t) \mathrm{d} \omega_{x}(L, t)+\left\langle y_{0}, \omega_{1}\right\rangle_{L^{\infty} \times X_{0}}-\left\langle y_{1}, \omega_{0}\right\rangle_{W^{-1, \infty} \times X_{1}} \\
\quad+\varepsilon\left(\int_{0}^{L} \operatorname{sign}(\mu(\cdot, T)) \mathrm{d} \omega(\cdot, T)+\int_{0}^{L} \operatorname{sign}\left(\mu_{t}(\cdot, T)\right) \mathrm{d} \omega_{t}(\cdot, T)\right)
\end{gathered}
$$

where $\omega$ denotes the solution of the adjoint system (32), (33), (34) with the initial data $\left(\omega_{0}, \omega_{1}\right)$. Define $I_{1}$ as in (39). Equation (48) implies the existence of a control $u=c^{2} I_{1} w_{1} \in L^{\infty}(0, T)$ that satisfies the inclusion

$$
u(t) \in I_{1} \operatorname{sign}\left(\mu_{x}(L, t)\right)
$$

and for all $\left(\omega_{0}, \omega_{1}\right) \in X_{1} \times X_{0}$ the equation

$$
\begin{aligned}
0=c^{2} \int_{0}^{T} u(t) \mathrm{d} \omega_{x}(L, t)+\left\langle y_{0}, \omega_{1}\right\rangle_{L \infty \times X_{0}} & -\left\langle y_{1}, \omega_{0}\right\rangle_{W^{-1, \infty} \times X_{1}} \\
& +\varepsilon\left(\int_{0}^{L} \operatorname{sign}(\mu(\cdot, T)) \mathrm{d} \omega(\cdot, T)+\int_{0}^{L} \operatorname{sign}\left(\mu_{t}(\cdot, T)\right) \mathrm{d} \omega_{t}(\cdot, T)\right)
\end{aligned}
$$

On the other hand, multiplying the state equation (15) (with the control $u$ ) by the solution $\omega$ of the adjoint system yields

$$
\begin{aligned}
0=c^{2} \int_{0}^{T} u(t) \mathrm{d} \omega_{x}(L, t)+\left\langle y_{0}, \omega_{1}\right\rangle_{L^{\infty} \times X_{0}}-\left\langle y_{1}, \omega_{0}\right\rangle_{W-1, \infty \times X_{1}} & \\
& -\left\langle y(\cdot, T), \omega_{t}(\cdot, T)\right\rangle_{L^{\infty} \times X_{0}}+\left\langle y_{t}(\cdot, T), \omega(\cdot, T)\right\rangle_{W^{-1, \infty} \times X_{1}}
\end{aligned}
$$

Combining these two identities (50), (51) yields

$$
\begin{aligned}
\left\langle y(\cdot, T), \omega_{t}(\cdot, T)\right\rangle_{L^{\infty} \times X_{0}}-\left\langle y_{t}(\cdot, T), \omega(\cdot, T)\right\rangle_{W^{-1, \infty} \times X_{1}} & \\
& =-\varepsilon\left(\int_{0}^{L} \operatorname{sign}\left(\mu(\cdot, T) \mathrm{d} \omega(\cdot, T)+\int_{0}^{L} \operatorname{sign}\left(\mu_{t}(\cdot, T)\right) \mathrm{d} \omega_{t}(\cdot, T)\right)\right.
\end{aligned}
$$


for all $\left(\omega_{0}, \omega_{1}\right) \in X_{1} \times X_{0}$. This implies the inequalities

$$
\|y(\cdot, T)\|_{\infty,(0, L)}=\sup _{\omega_{t}(\cdot, T) \in X_{0}} \frac{\left\langle y(\cdot, T), \omega_{t}(\cdot, T)\right\rangle_{L^{\infty} \times X_{0}}}{\left\|\omega_{t}(\cdot, T)\right\|_{X_{0}}} \leq \varepsilon \frac{\int_{0}^{L} \mathrm{~d}\left|\omega_{t}(T, \cdot)\right|}{\left\|\omega_{t}(\cdot, T)\right\|_{X_{0}}}=\varepsilon
$$

and

$$
\left\|y_{t}(\cdot, T)\right\|_{\infty,(0, L)}=\sup _{\omega(\cdot, T) \in X_{1}} \frac{\left\langle y_{t}(\cdot, T), \omega(\cdot, T)\right\rangle_{W^{-1, \infty} \times X_{1}}}{\|\omega(\cdot, T)\|_{X_{1}}} \leq \varepsilon \frac{\int_{0}^{L} \mathrm{~d}|\omega(T, \cdot)|}{\|\omega(\cdot, T)\|_{X_{0}}}=\varepsilon
$$

which means that $y$ satisfies the $\varepsilon$-approximate end conditions (44). Hence the control $u$ that satisfies (49) and (50) is feasible for $P(\varepsilon)$. As in (47), we see that

$$
\begin{aligned}
& \frac{1}{2}\|u\|_{\infty,(0, T)}^{2}-J\left(\left(\mu_{0}, \mu_{1}\right)\right)+\varepsilon\left(\|\mu(\cdot, T)\|_{X_{1}}+\left\|\mu_{t}(\cdot, T)\right\|_{X_{0}}\right) \\
= & \frac{c^{4}}{2} I_{1}^{2}-c^{4} \int_{0}^{T} I_{1} \operatorname{sign}\left(\mu_{x}(L, t)\right) \mathrm{d} \mu_{x}(L, t)+\frac{c^{4}}{2} I_{1}^{2} \\
& +\left\langle y(\cdot, T), \mu_{t}(\cdot, T)\right\rangle_{L^{\infty} \times X_{0}}-\left\langle y_{t}(\cdot, T), \mu(\cdot, T)\right\rangle_{W^{-1, \infty} \times X_{1}}+\varepsilon\left(\|\mu(\cdot, T)\|_{X_{1}}+\left\|\mu_{t}(\cdot, T)\right\|_{X_{0}}\right) \\
= & c^{4} I_{1}^{2}-c^{4} I_{1} \int_{0}^{T} \mathrm{~d}\left|\mu_{x}(L, t)\right| \\
& -\varepsilon\left(\int _ { 0 } ^ { L } \operatorname { s i g n } \left(\mu(\cdot, T) \mathrm{d} \mu(\cdot, T)+\int_{0}^{L} \operatorname{sign}\left(\mu_{t}(\cdot, T) \mathrm{d} \mu_{t}(\cdot, T)\right)+\varepsilon\left(\|\mu(\cdot, T)\|_{X_{1}}+\left\|\mu_{t}(\cdot, T)\right\|_{X_{0}}\right)=0 .\right.\right.
\end{aligned}
$$

This means that $P(\varepsilon)$ and $D U A L(\varepsilon)$ have the same optimal values and that the control $u$ that satisfies (49) and (50) solves $P(\varepsilon)$. Thus we have constructed an $L^{\infty}$ norm minimal control.

Note that (49) implies that

$$
u(t) \in\left\{-\|u\|_{\infty,(0, T)},\|u\|_{\infty,(0, T)}\right\} \text { for all } t \in \operatorname{supp}\left(\mu_{x}(L, \cdot)\right) .
$$

The weak bang-bang principle for $\varepsilon$-approximate optimal control has exactly the same form as for the corresponding problem $P=P(0)$ of exact optimal control.

The existence of a solution of problem $D U A L(\varepsilon)$ can be proved as in Section 5. The term $\varepsilon\left(\|\mu(\cdot, T)\|_{X_{1}}+\right.$ $\left.\left\|\mu_{t}(\cdot, T)\right\|_{X_{0}}\right)$ in the objective function of $D U A L(\varepsilon)$ can be considered as a regularization term.

\subsection{The optimal value function of $P(\varepsilon)$}

In this section we analyze the behaviour of the optimal value of $P(\varepsilon)$ as a function of $\varepsilon$. The behaviour at $\varepsilon=0$ is particularly interesting.

Theorem 6.1. Let $\omega(\varepsilon)$ denote the optimal value of $P(\varepsilon)$. Then the function $\omega:[0, \infty) \rightarrow \mathbf{R}$ is decreasing. Let $\left(\mu_{0}, \mu_{1}\right)$ denote a solution of DUAL(0) and $\mu$ the corresponding solution of the adjoint system (32)-(34).

Let $\left(\mu_{0}^{\varepsilon}, \mu_{1}^{\varepsilon}\right)$ denote a solution of DUAL( $\left.\varepsilon\right)$ and $\mu^{\varepsilon}$ the corresponding solution of the adjoint system (32)-(34). Then the following inequality holds:

$$
\omega(0)-\varepsilon\left(\|\mu(\cdot, T)\|_{X_{1}}+\left\|\mu_{t}(\cdot, T)\right\|_{X_{0}}\right) \leq \omega(\varepsilon) \leq \omega(0)-\varepsilon\left(\left\|\mu^{\varepsilon}(\cdot, T)\right\| X_{X_{1}}+\left\|\mu_{t}^{\varepsilon}(\cdot, T)\right\|_{X_{0}}\right) .
$$

In particular, $\omega$ is continuous at zero.

Proof. Let $0 \leq \varepsilon_{1}<\varepsilon_{2}$ be given. The feasible set of $P\left(\varepsilon_{1}\right)$ is contained in the feasible set of $P\left(\varepsilon_{2}\right)$, hence $\omega\left(\varepsilon_{2}\right) \leq \omega\left(\varepsilon_{1}\right)$. We insert the dual solution $\left(\mu_{0}, \mu_{1}\right)$ in the objective functional of $D U A L(\varepsilon)$. Then weak duality 
for $P(\varepsilon)$ and strong duality for $P(0)$ yield

$$
\begin{aligned}
\omega(\varepsilon) & \geq J\left(\mu_{0}, \mu_{1}\right)-\varepsilon\left(\|\mu(\cdot, T)\|_{X_{1}}+\left\|\mu_{t}(\cdot, T)\right\|_{X_{0}}\right) \\
& =\omega(0)-\varepsilon\left(\|\mu(\cdot, T)\|_{X_{1}}+\left\|\mu_{t}(\cdot, T)\right\|_{X_{0}}\right) .
\end{aligned}
$$

We insert the dual solution $\left(\mu_{0}^{\varepsilon}, \mu_{1}^{\varepsilon}\right)$ in the objective functional of $D U A L(0)$. Then weak duality for $P(0)$ and strong duality for $P(\varepsilon)$ yield

$$
\begin{aligned}
\omega(0) & \geq J\left(\mu_{0}^{\varepsilon}, \mu_{1}^{\varepsilon}\right)=J\left(\mu_{0}^{\varepsilon}, \mu_{1}^{\varepsilon}\right)-\varepsilon\left(\left\|\mu^{\varepsilon}(\cdot, T)\right\|_{X_{1}}+\left\|\mu_{t}^{\varepsilon}(\cdot, T)\right\|_{X_{0}}\right)+\varepsilon\left(\left\|\mu^{\varepsilon}(\cdot, T)\right\|_{X_{1}}+\left\|\mu_{t}^{\varepsilon}(\cdot, T)\right\|_{X_{0}}\right) \\
& =\omega(\varepsilon)+\varepsilon\left(\left\|\mu^{\varepsilon}(\cdot, T)\right\|_{X_{1}}+\left\|\mu_{t}^{\varepsilon}(\cdot, T)\right\|_{X_{0}}\right)
\end{aligned}
$$

and the assertion follows.

\subsection{An example for $p(\varepsilon)$ with explicit solution}

Results from [10] imply that for $T=4 L / c, y_{0}(x)=\sin (x \pi / L)$ and $y_{1}(x)=0$ the control

$$
u(t)=\sin (c t \pi / L) / 4
$$

is a solution of the optimization problem $P(0)$. Hence the optimal value of $P(0)$ is equal to $1 / 32$. In fact, with $t_{1}=x / c$ Theorem 2.1 yields

$$
y(x, T)=u\left(t_{0}+x / c\right)-u\left(t_{0}-x / c\right)+u\left(3 t_{0}+x / c\right)-u\left(3 t_{0}-x / c\right)+y_{0}(x)=0 .
$$

Moreover, we have

$$
y_{t}(x, T)=u^{\prime}\left(t_{0}+x / c\right)-u^{\prime}\left(t_{0}-x / c\right)+u^{\prime}\left(3 t_{0}+x / c\right)-u^{\prime}\left(3 t_{0}-x / c\right)=0
$$

The control $u$ is not bang-bang-off. In fact, Theorem 3.1 implies that no feasible bang-bang-off control exists.

The strong duality implies that $\left(\mu_{0}, \mu_{1}\right)=\left(0,-(1 / 16) \delta_{L / 2}(t)\right)$ is a solution of $D U A L(0)$.

For $\varepsilon \in[0,1]$, define the control function

$$
u_{\varepsilon}(t)=(1-\varepsilon) \sin (c t \pi / L) / 4=(1-\varepsilon) u(t)
$$

with $u$ from (53). Then we have

$$
\begin{aligned}
y(x, T) & =u\left(t_{0}+x / c\right)-u\left(t_{0}-x / c\right)+u\left(3 t_{0}+x / c\right)-u\left(3 t_{0}-x / c\right)+y_{0}(x) \\
& =\sin (x / L \pi)+\frac{1-\varepsilon}{4}[\sin (x \pi / L-\pi)+\sin (x \pi / L+\pi)]+\frac{1-\varepsilon}{4}[\sin (x \pi / L-3 \pi)+\sin (x \pi / L+3 \pi)] \\
& =\sin (x / L \pi)+\frac{1-\varepsilon}{2} \sin (x / L \pi) \cos (\pi)+\frac{1-\varepsilon}{2} \sin (x / L \pi) \cos (3 \pi)=\sin (x / L \pi)-(1-\varepsilon) \sin (x / L \pi) \\
& =\varepsilon \sin (x / L \pi) .
\end{aligned}
$$

Moreover, we have

$$
\begin{aligned}
y_{t}(x, T) & =u^{\prime}\left(t_{0}+x / c\right)-u^{\prime}\left(t_{0}-x / c\right)+u^{\prime}\left(3 t_{0}+x / c\right)-u^{\prime}\left(3 t_{0}-x / c\right)+y_{1}(x) \\
& =\frac{\varepsilon-1}{4} \frac{c \pi}{L}\left[\cos \left(\pi-\frac{x \pi}{L}\right)-\cos \left(\pi+\frac{x \pi}{L}\right)+\cos \left(3 \pi-\frac{x \pi}{L}\right)-\cos \left(3 \pi+\frac{x \pi}{L}\right)\right] \\
& =\frac{\varepsilon-1}{2} \frac{c \pi}{L}[\sin (\pi) \sin (x / L \pi)+\sin (3 \pi) \sin (x / L \pi)]=0 .
\end{aligned}
$$




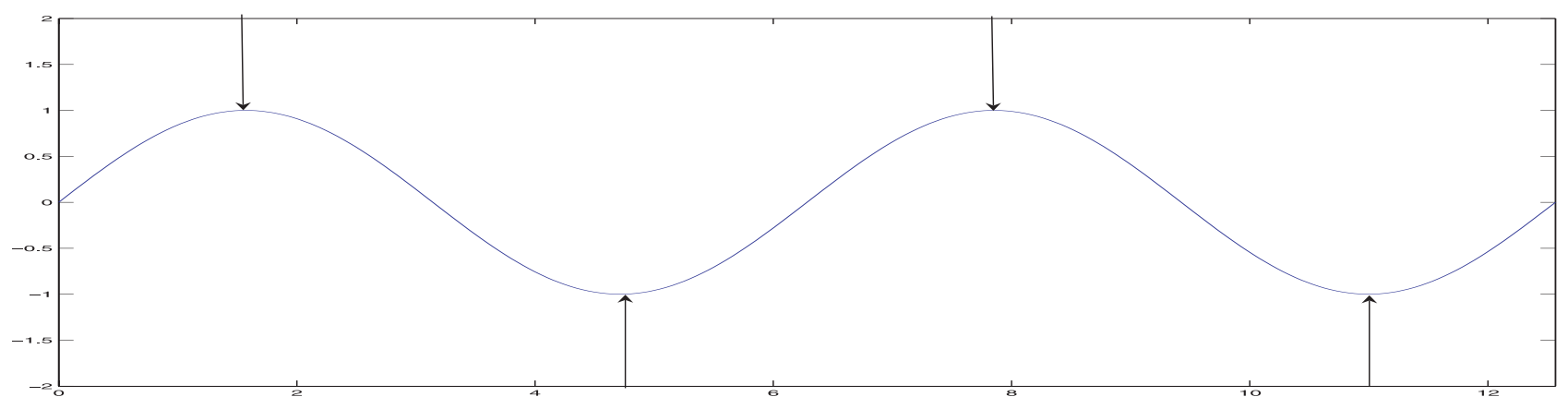

Figure 5. The optimal control $u_{0}(t)$ (Sect. 6.4).

So we see that $u_{\varepsilon}$ is feasible for problem $P(\varepsilon)$. This yields an upper bound for the optimal value $\omega(\varepsilon)$, namely

$$
\omega(\varepsilon) \leq\left\|u_{\varepsilon}\right\|_{\infty,(0, T)}^{2} / 2=(1-\varepsilon)^{2} / 32 .
$$

To obtain a lower bound for $\omega(\varepsilon)$, we look at a point which is feasible for the dual problem $D U A L(\varepsilon)$. Define the dual feasible point

$$
\left.\left(\mu_{0}^{\varepsilon}, \mu_{1}^{\varepsilon}\right)=(0,(\varepsilon-1) / 16) \delta_{L / 2}(t)\right)=(1-\varepsilon)\left(\mu_{0}, \mu_{1}\right) .
$$

Theorem 2.1 implies that for the solution $\mu^{\varepsilon}$ of the adjoint system $(32)-(34)$ we have $\mu^{\varepsilon}(\cdot, T)=\mu^{\varepsilon}(\cdot, 0)=\mu_{0}$ and $\mu_{t}^{\varepsilon}(\cdot, T)=\mu_{t}^{\varepsilon}(\cdot, 0)=\mu_{1}$. This implies $\left\|\mu^{\varepsilon}(\cdot, T)\right\|_{X_{1}}=0$ and $\left\|\mu_{t}^{\varepsilon}(\cdot, T)\right\|_{X_{0}}=(1-\varepsilon) / 16$. Moreover,

$$
-\left\langle y_{0}, \mu_{1}\right\rangle_{L \infty \times X_{0}}+\left\langle y_{1}, \mu_{0}\right\rangle_{W^{-1, \infty} \times X_{1}}=(1-\varepsilon) / 16
$$

and

$$
\int_{0}^{T} \mathrm{~d}\left|\mu_{x}(L, \cdot)\right|=\frac{4}{c^{2}} \int_{0}^{L} \mathrm{~d}\left|\mu_{1}\right|=\frac{1-\varepsilon}{4 c^{2}} .
$$

Hence for the value of the objective functional of $D U A L(\varepsilon)$ we have

$$
\begin{aligned}
J\left(\left(\mu_{0}^{\varepsilon}, \mu_{1}^{\varepsilon}\right)\right)-\varepsilon\left(\left\|\mu^{\varepsilon}(\cdot, T)\right\|_{X_{1}}+\left\|\mu_{t}^{\varepsilon}(\cdot, T)\right\|_{X_{0}}\right) & \\
& =-\frac{1}{2}\left[\frac{1-\varepsilon}{4}\right]^{2}+\frac{1-\varepsilon}{16}-\varepsilon \frac{1-\varepsilon}{16}=-\frac{(1-\varepsilon)^{2}}{32}+\frac{(1-\varepsilon)^{2}}{16}=\frac{(1-\varepsilon)^{2}}{32} .
\end{aligned}
$$

Now weak duality implies that $\omega(\varepsilon) \geq(1-\varepsilon)^{2} / 32$. With the upper bound (56) this yields $\omega(\varepsilon)=(1-\varepsilon)^{2} / 32$ which implies that $u_{\varepsilon}$ is a solution of $P(\varepsilon)$. Note that the optimal control $u^{\varepsilon}$ is not of bang-bang-off type.

Figure 5 shows the optimal control $u_{0}$. The arrows indicate the points where the weak bang-bang principle is a restriction on the control function. The direction of the arrows indicate the sign of $\mu_{x}(L, \cdot)$ : If the arrows point downwards, the sign is positive. If the arrows point upwards, the sign is negative. At the points on the time-axis where no arrow appears, $\mu_{x}(L, \cdot)$ is not supported, that is has the value zero.

Figure 6 shows an approximation of adjoint state $\mu$ generated by the dual solution $\left(\mu_{0}, \mu_{1}\right)=\left(0,-(1 / 16) \delta_{L / 2}(t)\right)$ of $D U A L(0)$ for $L=c=1$. For the exact solution, the flat surfaces are connected by jump discontinuities.

Figure 7 shows the system state $y$ generated by the control $u_{0}$ for $L=c=1$. Note that the state is continuous.

\subsubsection{An optimal approximate control with bang-intervals}

For $\varepsilon \in[0,1]$, define the set $\left.M_{\varepsilon}=\left\{t \in[0, T]: \mid u_{\varepsilon}(t)\right) \mid \geq\left\|u_{\varepsilon}\right\|_{\infty,(0, T)}(1-\varepsilon)\right\}$. Define the control function

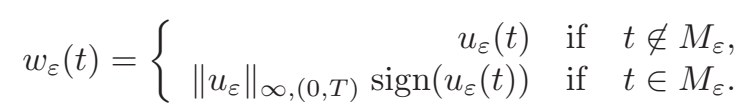




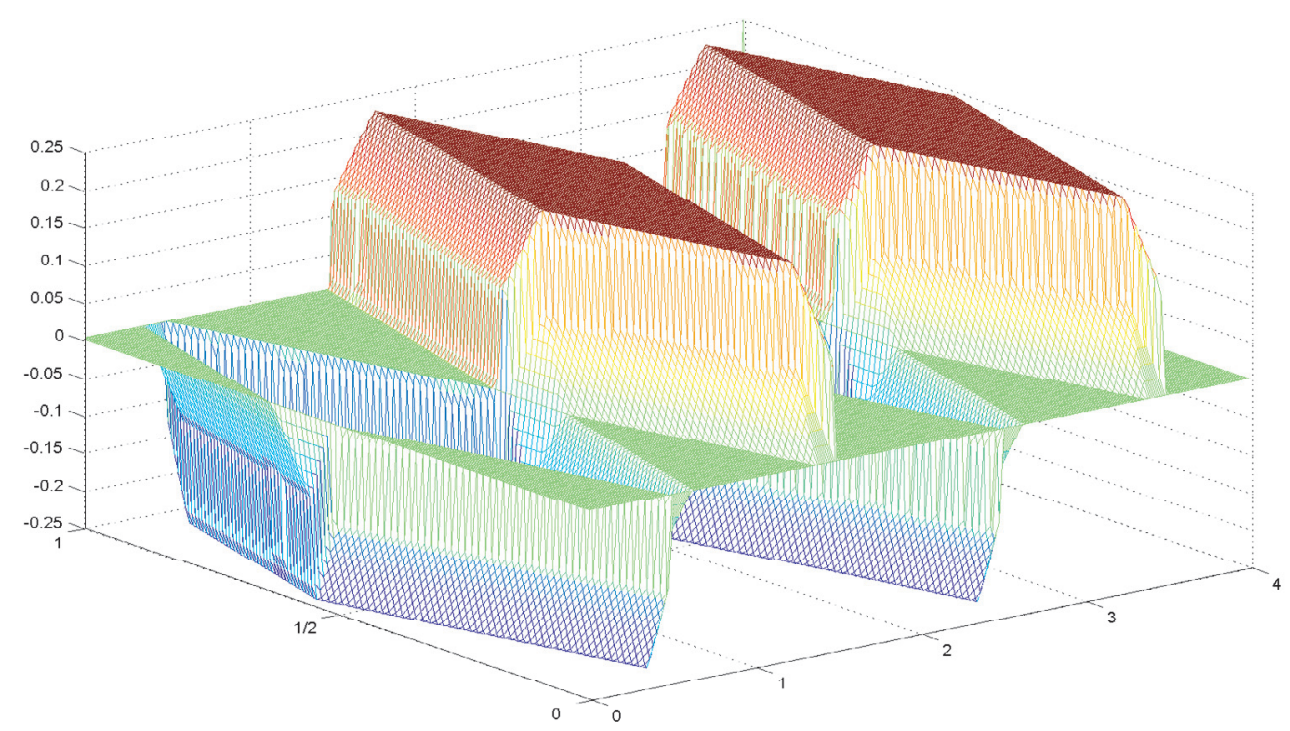

Figure 6. The adjoint state for $\varepsilon=0$ (Sect. 6.4).

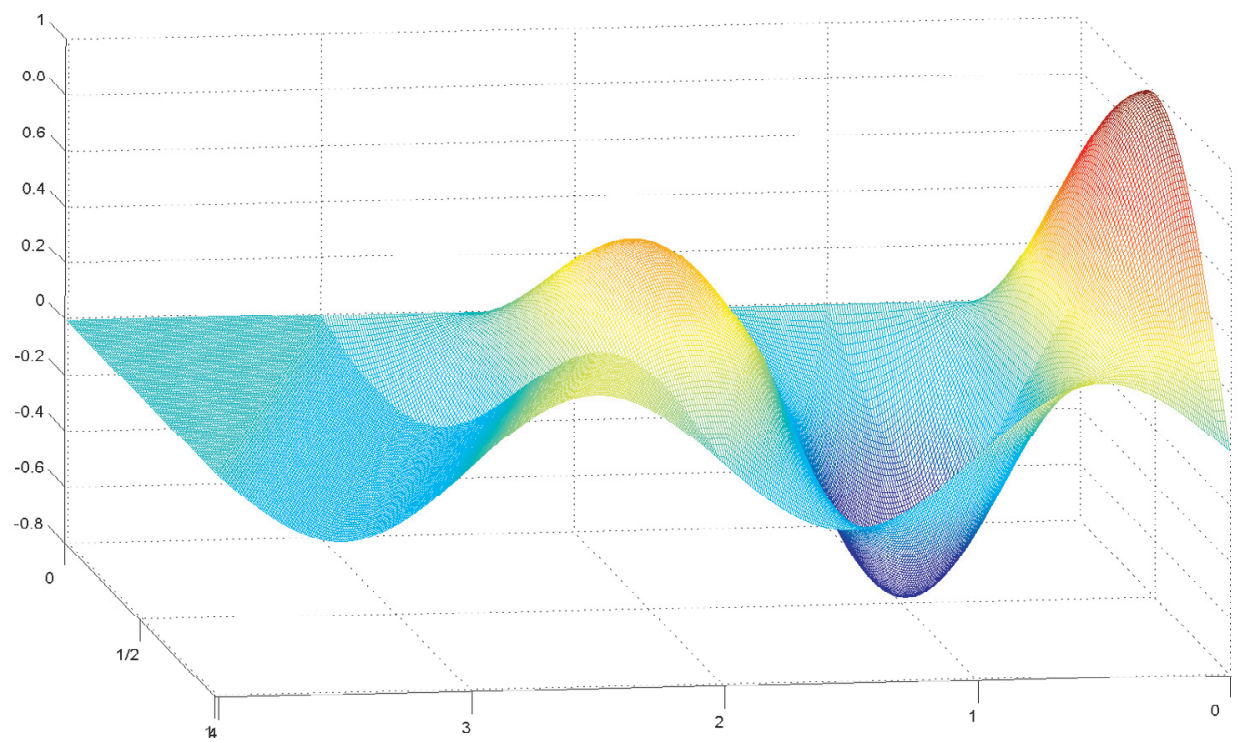

Figure 7 . The system state $y$ generated by $u_{0}$ (Sect. 6.4 ).

Then for the corresponding velocity we have $y_{t}(x, T)=0$.

If $t_{0}+x / c \notin M_{\varepsilon}$, we have also $t_{0}-x / c, 3 t_{0}+x / c, 3 t_{0}-x / c \notin M_{\varepsilon}$, which implies that $|y(x, T)|=\varepsilon|\sin (x \pi / L)|$ $\leq \varepsilon$. 
If $t_{0}+x / c \in M_{\varepsilon}$, we have also $t_{0}-x / c, 3 t_{0}+x / c, 3 t_{0}-x / c \in M_{\varepsilon}$, which implies that for the state corresponding to $w_{\varepsilon}$ we have

$$
\begin{gathered}
y(x, T)=y_{0}(x)-\left\|u_{\varepsilon}\right\|_{\infty,(0, T)}-\left\|u_{\varepsilon}\right\|_{\infty,(0, T)}-\left\|u_{\varepsilon}\right\|_{\infty,(0, T)}-\left\|u_{\varepsilon}\right\|_{\infty,(0, T)} \\
=\sin (x \pi / L)-(1-\varepsilon)=\varepsilon+\sin (x \pi / L)-1 \leq \varepsilon .
\end{gathered}
$$

If $t_{0}+x / c \in M_{\varepsilon}$, we have

$$
\sin (x \pi / L) \geq 1-\varepsilon
$$

This implies

$$
y(x, t) \geq \varepsilon-\varepsilon \geq 0 .
$$

So we have shown that $w_{\varepsilon}$ is a feasible control for $P(\varepsilon)$. Since $\left\|w_{\varepsilon}\right\|_{\infty,(0, T)}=\left\|u_{\varepsilon}\right\|_{\infty,(0, T)}$, this implies that $w_{\varepsilon}$ is a solution of $P(\varepsilon)$ with bang-intervals.

For $\varepsilon=1$, we obtain a pure bang-bang control. As $\varepsilon$ tends to zero, the length of the bang-intervals also converges to zero. Figure 8 shows the optimal control $w_{\varepsilon}$ for $L=c=1$ and $\varepsilon=0.1$.

\subsubsection{Nonexistence of dual solutions given as Lebesgue integrable functions}

In this section we want to analyze whether it is possible that for our example problem regular dual solutions exist that are given by integrable functions and where no measures appear.

Assume that $\left(\mu_{0}, \mu_{1}\right)$ is a solution of problem $D U A L(\varepsilon)$ for our example and $\mu$ is the solution of the adjoint system (32)-(34). Let $y$ denote the state that is generated by the optimal control $u_{\varepsilon}$. Equation (51) yields

$$
\begin{aligned}
\int_{0}^{L} \sin (x \pi / L) \mathrm{d} \mu_{1}(x) & =\left\langle y_{0}, \mu_{1}\right\rangle_{L^{\infty} \times X_{0}}-\left\langle y_{1}, \mu_{0}\right\rangle_{W^{-1, \infty} \times X_{1}} \\
& =-c^{2} \int_{0}^{T} u_{\varepsilon}(t) \mathrm{d} \mu_{x}(L, t)+\left\langle y(\cdot, T), \mu_{t}(\cdot, T)\right\rangle_{L^{\infty} \times X_{0}}-\left\langle y_{t}(\cdot, T), \mu(\cdot, T)\right\rangle_{W^{-1, \infty} \times X_{1}} \\
& =-c^{2} \int_{0}^{T} u_{\varepsilon}(t) \mathrm{d} \mu_{x}(L, t)+\int_{0}^{L} \varepsilon \sin (x \pi / L) \mathrm{d} \mu_{t}(x, T)
\end{aligned}
$$

Since $T=4 L / c$, we have $\mu_{t}(\cdot, T)=\mu_{t}(\cdot, 0)=\mu_{1}$, hence the above equation yields

$$
(1-\varepsilon) \int_{0}^{L} \sin (x \pi / L) \mathrm{d} \mu_{1}(x)=-c^{2} \int_{0}^{T} u_{\varepsilon}(t) \mathrm{d} \mu_{x}(L, t) .
$$

Suppose that $\mu_{1}$ is a measure that is supported in other points than $L / 2$. Then we have

$$
\int_{0}^{T} u_{\varepsilon}(t) \mathrm{d} \mu_{x}(L, t)=\int_{0}^{T}(1-\varepsilon) \frac{\sin (c t \pi / L)}{4} \mathrm{~d} \mu_{x}(L, t)<\frac{1-\varepsilon}{4} \int_{0}^{T} \mathrm{~d}\left|\mu_{x}(L, t)\right| .
$$

Therefore in this case (58) yields the strict inequality

$$
(1-\varepsilon) \int_{0}^{L}-\sin (x \pi / L) \mathrm{d} \mu_{1}(x)=c^{2} \int_{0}^{T} u_{\varepsilon}(t) \mathrm{d} \mu_{x}(L, t)<c^{2} \frac{1-\varepsilon}{4} \int_{0}^{T} \mathrm{~d}\left|\mu_{x}(L, t)\right|=\frac{1-\varepsilon}{4} c^{2} I_{1} .
$$

The weak bang-bang principle (49) implies that

$$
\frac{1-\varepsilon}{4}=\left\|u_{\varepsilon}\right\|_{\infty,(0, T)}=c^{2} I_{1}
$$


The objective function of $D U A L(\varepsilon)$ is

$$
\begin{gathered}
J\left(\left(\mu_{0}, \mu_{1}\right)\right)-\varepsilon\left(\|\mu(\cdot, T)\|_{X_{1}}+\left\|\mu_{t}(\cdot, T)\right\|_{X_{0}}\right) \\
=-c^{4} \frac{I_{1}^{2}}{2}-\left\langle y_{0}, \mu_{1}\right\rangle_{L^{\infty} \times X_{0}}+\left\langle y_{1}, \mu_{0}\right\rangle_{W^{-1, \infty} \times X_{1}}-\varepsilon\left(\|\mu(\cdot, T)\|_{X_{1}}+\left\|\mu_{t}(\cdot, T)\right\|_{X_{0}}\right) \\
=-c^{4} \frac{I_{1}^{2}}{2}-\left\langle y_{0}, \mu_{1}\right\rangle_{L^{\infty} \times X_{0}}-\varepsilon\left(\left\|\mu_{0}\right\|_{X_{1}}+\left\|\mu_{1}\right\|_{X_{0}}\right)
\end{gathered}
$$

because on account of $T=4 L / c$, we have $\mu(\cdot, T)=\mu(\cdot, 0)$. The $\varepsilon$-approximate end conditions (44) implies that

$$
\begin{gathered}
-\varepsilon\left(\left\|\mu_{0}\right\|_{X_{1}}+\left\|\mu_{1}\right\|_{X_{0}}\right) \leq\left\langle y(\cdot, T), \mu_{1}\right\rangle_{L^{\infty} \times X_{0}}+\left\langle y_{t}(\cdot, T), \mu_{0}\right\rangle_{W^{-1, \infty} \times X_{1}} \\
=\left\langle y(\cdot, T), \mu_{1}\right\rangle_{L^{\infty} \times X_{0}}=\int_{0}^{L} \varepsilon \sin (x \pi / L) \mathrm{d} \mu_{1}(x) .
\end{gathered}
$$

Therefore we can conclude that if $\mu_{1}$ is supported in other points than $L / 2$, (59) yields the strict inequality

$$
\begin{gathered}
J\left(\left(\mu_{0}, \mu_{1}\right)\right)-\varepsilon\left(\|\mu(\cdot, T)\|_{X_{1}}+\left\|\mu_{t}(\cdot, T)\right\|_{X_{0}}\right) \\
=-c^{4} \frac{I_{1}^{2}}{2}-\int_{0}^{L} \sin (x \pi / L) \mathrm{d} \mu_{1}(x)-\varepsilon\left(\|\mu(\cdot, T)\|_{X_{1}}+\left\|\mu_{t}(\cdot, T)\right\|_{X_{0}}\right) \\
\leq-c^{4} \frac{I_{1}^{2}}{2}-\int_{0}^{L} \sin (x \pi / L) \mathrm{d} \mu_{1}(x)+\int_{0}^{L} \varepsilon \sin (x \pi / L) \mathrm{d} \mu_{1}(x) \\
=-c^{4} \frac{I_{1}^{2}}{2}+(1-\varepsilon) \int_{0}^{L}-\sin (x \pi / L) \mathrm{d} \mu_{1}(x) \\
\quad<-c^{4} \frac{I_{1}^{2}}{2}+\frac{1-\varepsilon}{4} c^{2} I_{1}=-\frac{(1-\varepsilon)^{2}}{32}+\frac{(1-\varepsilon)^{2}}{16}=\frac{(1-\varepsilon)^{2}}{32}=\omega(\varepsilon)
\end{gathered}
$$

where as in Section 6.3, $\omega(\varepsilon)$ denotes the optimal value of $D U A L(\varepsilon)$.

So we see that there exists no dual solution where $\mu_{1}$ is not given by a Dirac measure.

\subsection{Relaxation with Hilbert space norms}

As an alternative to the problem of optimal $\varepsilon$-approximate control $P(\varepsilon)$ defined in Section 6.1 we consider a problem where the end conditions are relaxed with respect to a Hilbert space norm, namely

$$
\begin{aligned}
Q(\varepsilon): \min (1 / 2)\|u\|_{\infty,(0, T)}^{2} \quad \text { such that } & (15),(16),(17) \text { and } \\
& \int_{0}^{L}|y(x, T)|^{2} \mathrm{~d} x+\left\|y_{t}(\cdot, T)\right\|_{H^{-1}(0, L)}^{2} \leq \varepsilon^{2}
\end{aligned}
$$

for $\varepsilon \geq 0$. Problem $Q(0)$ is equivalent to the problem $P$ of $L^{\infty}$-norm minimal exact control defined above.

In contrast to the relaxed problem $P(\varepsilon)$ that is defined above in a non-reflexive setting, for $\varepsilon>0$ problem $Q(\varepsilon)$ yields controls with bang-intervals. This is illustrated by an example in the next section.

\subsubsection{Example for relaxation with Hilbert space norms}

Consider our example with $T=4 L / c, y_{0}(x)=\sin (x \pi / L)$ and $y_{1}(x)=0$. For $\delta \in(0,1)$ define the control

$$
u(t)=\left\{\begin{array}{rll}
\sin (c t \pi / L) / 4 & \text { if } & |\sin (c t \pi / L)| / 4 \leq(1-\delta) / 4 \\
(1-\delta) / 4 \operatorname{sign}(\sin (c t \pi / L)) & \text { if } & |\sin (c t \pi / L)| / 4>(1-\delta) / 4
\end{array}\right.
$$

Then $u$ has four bang-intervals. 


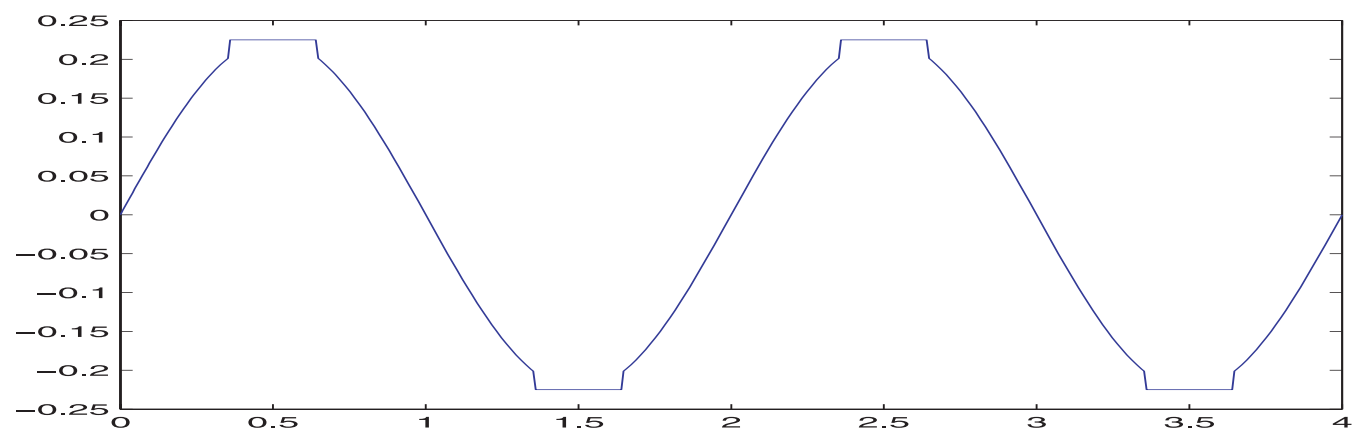

Figure 8. The optimal approximate control $w_{\varepsilon}$ with bang-intervals (Sect. 6.4.1).

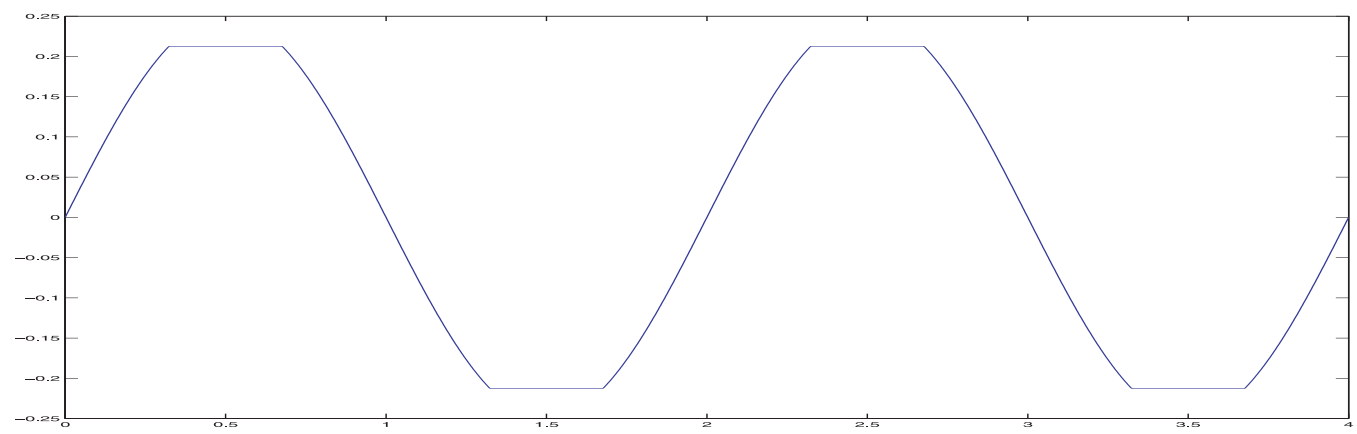

FIGURE 9. Hilbert space relaxation: the optimal control $u$ with bang-intervals (Sect. 6.5).

Figure 9 shows the control $u$ for $L=c=1$ and $\delta=0.15$.

Equation (55) implies that the control $u$ generates a state with $y_{t}(\cdot, T)=0$. Moreover, by $(54)$ we have

$$
y(x, T)=\left\{\begin{array}{rll}
0 & \text { if } & \sin (x \pi / L) \leq(1-\delta), \\
\sin (x \pi / L)-(1-\delta) & \text { if } \quad \sin (x \pi / L)>(1-\delta) .
\end{array}\right.
$$

Figure 10 shows the terminal state $y(\cdot, T)$ for $L=c=1$ and $\delta=0.15$. Note that $y(\cdot, T)$ is positive and thus can be seen as the density of a measure, which appears also in the corresponding necessary optimality conditions. The support of $y(\cdot, T)$ corresponds to the bang-intervals of the control $u$. In fact, the form of $y(\cdot, T)$ implies that $u$ satisfies the necessary optimality condition for $Q(\varepsilon)$ where $\varepsilon$ is defined as a function of $\delta$ by the equation

$$
\varepsilon^{2}=\int_{0}^{L} y(x, T)^{2} \mathrm{~d} x .
$$

The necessary optimality conditions for $Q(\varepsilon)$ are satisfied if a Lagrange multiplier $\lambda \in[0, \infty)$ and a nonnegative measure $\mu$ exist such that for all $v \in L^{\infty}(0, T)$ the following equation holds:

$$
0=\int_{0}^{T} \operatorname{sign}(u(t)) v(t) \mathrm{d} \mu(t)+2 \lambda \int_{0}^{L} y(x, T)\left[v\left(t_{0}+x / c\right)-v\left(t_{0}-x / c\right)+v\left(3 t_{0}+x / c\right)-v\left(3 t_{0}-x / c\right)\right] \mathrm{d} x
$$

Let $I_{b} \subset[0, T]$ denote the set of bang-points of $u$. We define a measure $\mu$ with support $I_{b}$ by

$$
\mathrm{d} \mu(t)=[|\sin (x \pi / L)|-(1-\delta)] \mathrm{d} t
$$




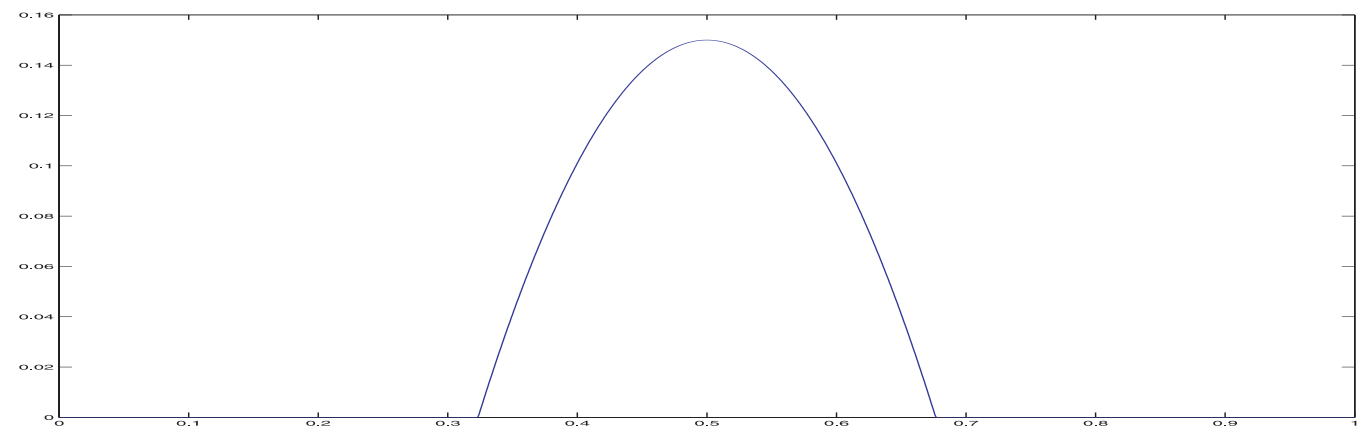

Figure 10. Hilbert space relaxation: the terminal state $y(\cdot, T)$ is the density of a measure (Sect. 6.5).

on $I_{b}$ and $\mu=0$ otherwise. For all $v \in L^{\infty}(0, T)$ we get

$$
\begin{aligned}
& \int_{0}^{T} \operatorname{sign}(u(t)) v(t) \mathrm{d} \mu(t) \\
& =\int_{0}^{L} y(x, T) v\left(t_{0}-x / c\right) \mathrm{d} x-\int_{0}^{L} y(x, T) v\left(t_{0}+x / c\right) \mathrm{d} x+\int_{0}^{L} y(x, T) v\left(3 t_{0}-x / c\right) \mathrm{d} x-\int_{0}^{L} y(x, T) v\left(3 t_{0}+x / c\right) \mathrm{d} x .
\end{aligned}
$$

Hence with the choice $\lambda=1 / 2$ equation (60) holds which implies that $u$ is an optimal $\varepsilon$-approximate control for the Hilbert space relaxation.

Since for $\varepsilon>0$, the solutions of $Q(\varepsilon)$ have bang-intervals, as in the example for the corresponding dual problem we find solutions that are measures with densities, that is solutions that can be interpreted as functions.

\section{Time-Optimal CONTROL}

The problem of time-optimal control is to find a control function that steers the system in minimal time exactly to the desired target state subject to an upper bound on the norm of the control function, in our case a bound on the $L^{\infty}$-norm. If this upper bound is sufficiently large, the minimal control time will in general be equal to the minimal time where exact controllability is possible, that is in our case twice the characteristic time $L / c$ that the characteristic curves need to travel from one end of the string to the other. When the upper bound is decreased, the corresponding minimal control time increases. In fact, there is a certain duality between the problem of time optimal control with a fixed upper bound and the problem of norm minimal control with a fixed control time. We will describe this relation in the next section.

\subsection{Duality between time optimal and norm minimal control}

For a given bound $\beta>0$, the problem of time optimal control corresponding to our system is

$$
\begin{aligned}
P_{0}(\beta): \min T \text { s.t. } & (15),(16),(17), \\
& y(x, T)=y_{t}(x, T)=0 \text { and } \\
& \frac{1}{2}\|u\|_{\infty,(0, T)}^{2} \leq \beta .
\end{aligned}
$$

Let $\tau(\beta)$ denote the minimal control time.

For a control time $T>0$, we define the problem of norm-minimal control

$$
\begin{aligned}
P(T): \min (1 / 2)\|u\|_{\infty,(0, T)}^{2} \text { s.t. } & (15),(16),(17) \text { and } \\
& y(x, T)=y_{t}(x, T)=0 .
\end{aligned}
$$


Let $\omega(T)$ denote the optimal value of problem $P(T)$. Then the function $\omega$ is decreasing.

We have the duality relation

$$
\tau(\beta)=\min \{T: \omega(T) \leq \beta\} .
$$

If the function $\omega$ is strictly decreasing and continuous (the so called normal case) the equation

$$
\omega(T)=\beta
$$

already determines $\tau(\beta)$ (see [14]) if $\beta$ is less than or equal to $\omega(2 L / c)$.

\subsection{An example for the non-normal case}

In [10] a norm minimal control problem for the wave equation with Dirichlet boundary controls at both ends is solved, also for the $L^{\infty}$ case, namely the problem

$$
\begin{array}{ll}
P(T): \quad & \min \max \left\{\left\|u_{-1}\right\|_{\infty,(0, T)},\left\|u_{1}\right\|_{\infty,(0, T)}\right\} \text { s.t. } \\
& y_{t t}(x, t)=c^{2} y_{x x}(x, t),(x, t) \in(0, L) \times(0, T) \\
& y(x, 0)=0, y_{t}(x, 0)=0, x \in(0, L) \\
& y(0, t)=u_{-1}(t), y(L, t)=u_{1}(t), t \in(0, T) \text { and } \\
& y(x, T)=y_{0}, y_{t}(x, T)=y_{1}, x \in(0, L) .
\end{array}
$$

In this case the minimal time where exact controllability holds is $L / c$. Let $\omega(T)$ denote the optimal value of problem $P(T)$ and $Y_{1}(x)=\int_{0}^{x} y_{1}(t) \mathrm{d} t$. Define

$$
\begin{array}{lc}
g_{1}(t)= & y_{0}(c t) / 2-(1 /(2 c)) Y_{1}(c t), \\
g_{2}(t)= & y_{0}(L-c t) / 2+(1 /(2 c)) Y_{1}(L-c t) .
\end{array}
$$

Then we have $\omega(T)=h_{\infty}\left(r_{0}\right)$, where $r_{0}$ is the real number that minimizes the function

$$
\begin{gathered}
h_{\infty}(r)=\max \left[\left\|\left(g_{1}(t)+r\right) /(k+1)\right\|_{\infty,(0, T-k L / c)},\left\|\left(g_{2}(t)-r\right) /(k+1)\right\|_{\infty,(0, T-k L / c)},\right. \\
\left.\left\|\left(g_{1}(t)+r\right) / k\right\|_{\infty,(T-k L / c, L / c)},\left\|\left(g_{2}(t)-r\right) / k\right\|_{\infty,(T-k L / c, L / c)}\right] .
\end{gathered}
$$

Here, $k$ is the natural number such that $k L / c \leq T<(k+1) L / c$.

As an example we consider $L=c=1$ and $y_{0}(t)=2$ if $t \in[0,1 / 2], y_{0}(t)=0$ if $t \in(1 / 2,1]$ and $y_{1}(t)=0$. Then we have $g_{1}(t)=1$ if $t \in[0,1 / 2], g_{1}(t)=0$ if $t \in(1 / 2,1]$ and $g_{2}(t)=0$ if $t \in[0,1 / 2), g_{2}(t)=1$ if $t \in[1 / 2,1]$.

If $T-k L / c \in[1 / 2,1]$ this yields

$$
\min _{r} h_{\infty}(r)=\min _{r} \max \left\{\frac{|1+r|}{k+1}, \frac{|r|}{k+1}, \frac{|r|}{k}, \frac{|1-r|}{k+1}, \frac{|1-r|}{k}\right\}=h_{\infty}\left(\frac{1}{2 k+1}\right)=\frac{1}{k+\frac{1}{2}} .
$$

If $T-k L / c \in[0,1 / 2)$ this yields

$$
\min _{r} h_{\infty}(r)=\min _{r} \max \left\{\frac{|1+r|}{k+1}, \frac{|1+r|}{k}, \frac{|r|}{k}, \frac{|r|}{k+1}, \frac{|r|}{k}, \frac{|1-r|}{k}\right\}=h_{\infty}(0)=\frac{1}{k} .
$$

This implies that the function $\omega$ is piecewise constant on intervals of length $1 / 2$ with jumps at the points $\{k, k+1 / 2, k \geq 1\}$. So here we are in the nonnormal case where the minimal control times only attain the discrete values $\{k, k+1 / 2, k \geq 1\}$. Figure 11 shows a plot of $\omega$.

Note that this nonnormal behaviour occurs only in the $L^{\infty}$-case. For the $L^{p}$ case with $p \in[2, \infty)$, the corresponding function $h_{p}(r)$ is of integral type and the situation is different: $\omega$ is continuous and strictly decreasing. This also follows from the computation of $\omega(T)$ in [10].

Continuity, Lipschitz and Hölder conditions for $\omega$ in the $L^{2}$ case are studied in [8]. 


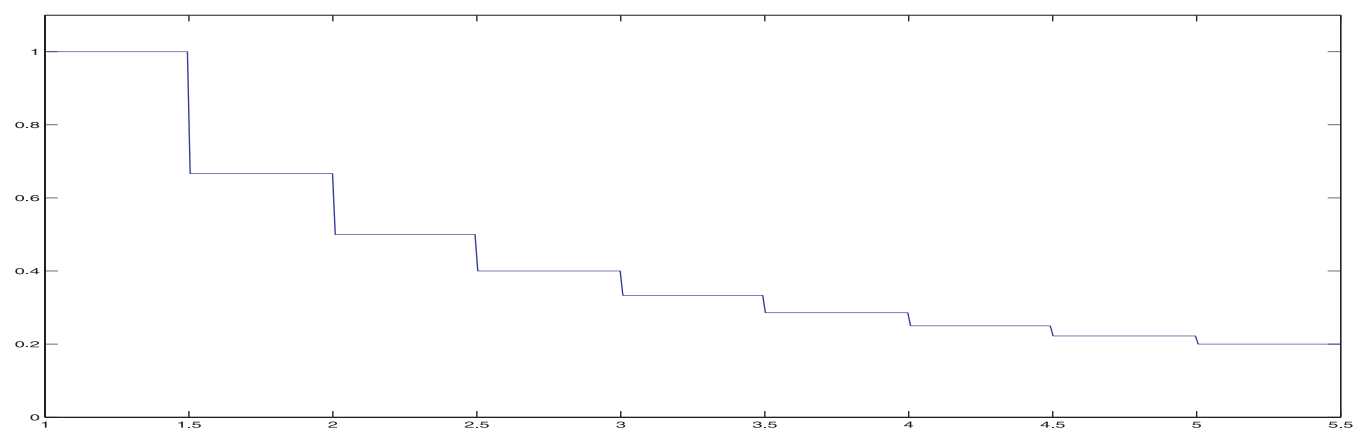

Figure 11. Time optimal control: the optimal value function $\omega(t)$ is discontinuous.

\section{Conclusion}

The purpose of this paper is to point out the fact that the weak forms of the bang-bang principle which hold for $L^{\infty}$-norm minimal control of the wave equation do not impose any restriction on the form of the optimal controls. The situation is similar for the problem of optimal exact control and optimal approximate control. For optimal exact control the weak bang-bang principle is given in (40) and for optimal approximate control in (49).

This situation is due to the fact that the dual solutions are in general given as measures, and it can happen that the support of the measures consist of a finite number of points only. Thus the set of points where these measures are zero can be quite large: This set is equal to the set where (40) ((49) respectively) is no restriction on the control values. We have given examples where the dual solutions are given by a Dirac measure.

Moreover, we have shown that the set of states that can be reached with bang-bang-off controls is quite small.

This situation has important consequences for numerical methods based upon the optimization of a discretized system: If the optimal controls of the discretized system are of bang-bang type (which may happen if the discretized system is governed by an ordinary differential equation) in general only weak convergence to the optimal control for the original system is possible. In [9], a method is described that takes into account this difficulty.

For the problem of time-optimal control with an $L^{\infty}$-norm bound for the controls, we have given an example for non-normal behaviour where the minimal control time as functions of the upper bound only attains values on a discrete grid.

Acknowledgements. We gratefully acknowledge fruitful disscussions on the subject with E. Zuazua at the Benasque Center for Science.

\section{REFERENCES}

[1] A. Barvinok, A course in convexity. AMS, Providence, Rhode Island (2002).

[2] J.K. Bennighof and R.L. Boucher, Exact minimum-time control of a distributed system using a traveling wave formulation. $J$. Optim. Theory Appl. 73 (1992) 149-167.

[3] F.H. Clarke, Optimization and Nonsmooth Analysis. John Wiley, New York (1983).

[4] A. Dovretzki, On Liapunov's convexity theorem. Proc. Natl. Acad. Sci. 91 (1994) 2145.

[5] V. Drobot, An infinte-dimensional version of Liapunov's convexity theorem. Michigan Math. J. 17 (1970) 405-408.

[6] C. Fabre, J.-P. Puel and E. Zuazua, Contrôlabilité approchée de l'équation de la chaleur linéaire avec des contrôles de norme $l^{\infty}$ minimale. (Approximate controllability for the linear heat equation with controls of minimal $l^{\infty}$ norm). C. R. Acad. Sci., Paris, Sér. I 316 (1993) 679-684.

[7] C. Fabre, J.-P. Puel and E. Zuazua, Approximate controllability of the semilinear heat equation. Proc. R. Soc. Edinb., Sect. A 125 (1995) 31-61. 
[8] M. Gugat, Time-parametric control: Uniform convergence of the optimal value functions of discretized problems. Contr. Cybern. 28 (1999) 7-33.

[9] M. Gugat and G. Leugering, Regularization of $l^{\infty}$-optimal control problems for distributed parameter systems. Comput. Optim. Appl. 22 (2002) 151-192.

[10] M. Gugat, G. Leugering and G. Sklyar, $l^{p}$-optimal boundary control for the wave equation. SIAM J. Control Optim. 44 (2005) 49-74.

[11] H. Hermes and J. Lasalle. Functional analysis and time optimal control. Academic Press (1969).

[12] T. Kato, Linear evolution equations of hyperbolic type. Univ. Tokyo Sec. I 17 (1970) 241-258.

[13] T. Kato, Perturbation theory for linear operators, Corr. printing of the 2nd edn. Springer (1980).

[14] W. Krabs, On moment theory and controllability of one-dimensional vibrating systems and heating processes, Lecture Notes in Control and Information Science 173. Springer-Verlag, Heidelberg (1992).

[15] W. Krabs, Optimal Control of Undamped Linear Vibrations. Heldermann Verlag, Lemgo, Germany (1995).

[16] C.M. Lee and F.D.K. Roberts, A comparison of algorithms for rational $l_{\infty}$ approximation. Math. Comp. 27 (1973) $111-121$.

[17] E.B. Lee and L. Markus, Foundations of Optimal Control Theory. Wiley, New York (1968).

[18] J.-L. Lions, Exact controllability, stabilization and perturbations of distributed systems. SIAM Rev. 30 (1988) 1-68.

[19] A. Lyapunov, Sur les fonctions-vecteurs complètement additives. Bull. Acad. Sci. URSS, Sér. Math. 4 (1940) 465-478.

[20] J. Macki and A. Strauss, Introduction to Optimal Control Theory. Springer-Verlag, New York (1982).

[21] V.J. Mizel and T.I. Seidman, An abstract bang-bang principle and time-optimal boundary control of the heat equation. SIAM J. Control Optim. 35 (1997) 1204-1216.

[22] N. Papageorgiu, Measurable multifunctions and their applications to convex integral functionals. Internat. J. Math. Math. Sciences 12 (1989) 175-192.

[23] G.K. Pedersen, Analysis Now. Springer-Verlag, New York (1989).

[24] R.T. Rockafellar, Convex Analysis. Princeton University Press (1970).

[25] K. Yosida, Functional Analysis. Springer, Berlin (1965).

[26] E. Zuazua, Optimal and approximate control of finite-difference approximation schemes for the 1d wave equation. Rend. Mat. Appl. 24 (2004) 201-237.

[27] E. Zuazua, Propagation, observation. and control of waves approximated by finite difference methods. SIAM Rev. 47 (2005) 197-243.

[28] E. Zuazua, Controllability of partial differential equations: Some results and open problems, in Handbook of Differential Equations: Evolutionary Differential Equations, C. Dafermos and E. Feireisl Eds., Elsevier Science (2006). 